\title{
Part III, Free Actions of Compact Quantum Groups on $\mathrm{C}^{*}$-Algebras
}

\author{
Kay SCHWIEGER ${ }^{\dagger}$ and Stefan WAGNER ${ }^{\ddagger}$ \\ $\dagger$ Iteratec GmbH, Stuttgart, Germany \\ E-mail: kay.schwieger@gmail.com \\ $\ddagger$ Blekinge Tekniska Högskola, Sweden \\ E-mail: stefan.wagner@bth.se
}

Received April 05, 2017, in final form August 05, 2017; Published online August 09, 2017

https://doi.org/10.3842/SIGMA.2017.062

\begin{abstract}
We study and classify free actions of compact quantum groups on unital $\mathrm{C}^{*}$ algebras in terms of generalized factor systems. Moreover, we use these factor systems to show that all finite coverings of irrational rotation $\mathrm{C}^{*}$-algebras are cleft.
\end{abstract}

Key words: free action; $\mathrm{C}^{*}$-algebra; quantum group; factor system; finite covering

2010 Mathematics Subject Classification: 46L85; 37B05; 55R10; 16D70

\section{Introduction}

Free actions of classical groups on $\mathrm{C}^{*}$-algebras were first introduced under the name saturated actions by Rieffel [26] (see also [21, 22]) and equivalent characterizations where given by Ellwood [10] and by Gottman, Lazar, and Peligrad [13, 20] (see also [3]). This class of actions does not admit degeneracies that may be present in general actions. For this reasons they are easier to understand and to classify. Indeed, for compact Abelian groups, free and ergodic actions, i.e., free actions with trivial fixed point algebra, were completely classified by Olesen, Pedersen and Takesaki in [19] and independently by Albeverio and Høegh-Krohn in [1]. This classification was generalized to compact non-Abelian groups by the remarkable work of Wassermann [31, 32, 33]. According to $[1,19,32]$, for a compact group $G$ there is a 1-to-1 correspondence between free and ergodic actions of $G$ and unitary 2-cocycles of the dual group. An analogous result in the context of compact quantum groups has been obtained by Bichon, De Rijdt and Vaes [4]. Extending this classification beyond the ergodic case is however not straightforward because, even for a commutative fixed point algebra, the action cannot necessarily be decomposed into a bundle of ergodic actions.

The study of non-ergodic free actions is also motivated by their role as noncommutative principal bundles in noncommutative geometry. In fact, by a classical result, having a free action of a compact group $G$ on a locally compact space $P$ is equivalent saying that $P$ carries the structure of a principal bundle over the quotient $X:=P / G$ with structure group $G$. Moreover, Rieffel showed that there is a 1-to-1 correspondence between classical free actions of compact groups on locally compact spaces and free actions of compact groups on commutative $\mathrm{C}^{*}$-algebras (cf. [21, Proposition 7.1.12 and Theorem 7.2.6]). From this perspective, the notion of a free action on a $\mathrm{C}^{*}$-algebra provides a natural framework for noncommutative principal bundles, which become increasingly prevalent in application to geometry and physics. Regarding classification, the case of locally trivial principal bundles, that is, if $P$ is glued together from spaces of the form $U \times G$ with an open subset $U \subseteq X$, is very well-understood. This gluing immediately leads to $G$-valued cocycles. The corresponding cohomology theory, called Čech cohomology, gives 
a complete classification of locally trivial principal bundles with base space $X$ and structure group $G$.

The present paper is a sequel of [27] and [28], where we studied free actions of compact Abelian groups and so-called cleft actions, respectively. To be more precise, we achieved in [27] a complete classification of free, but not necessary ergodic actions of compact Abelian groups on unital $\mathrm{C}^{*}$-algebras. This classification extends the results of $[1,19]$ and relies on the fact that the corresponding isotypic components are Morita self-equivalence over the fixed point algebra. Moreover, we provided a classification of principal bundles with compact Abelian structure group which are not locally trivial. For free actions of non-Abelian compact groups the bimodule structure of the corresponding isotypic components is more subtle. For this reason we concentrated in [28] on a simple class of free actions of non-Abelian compact groups, namely cleft actions. Regarded as noncommutative principal bundles, these actions are characterized by the fact that all associated noncommutative vector bundles are trivial. In the present article we turn to the general case of free actions of compact quantum groups. The main objective of this article is to provide a complete description of free actions of compact quantum groups on unital $\mathrm{C}^{*}$-algebras in terms of so-called factor systems. Besides an interesting characterization of freeness, our approach uses the fact that nonergodic actions of compact quantum groups can be described in terms of weak unitary tensor functors, i.e., functors from the representation category of the underlying compact quantum group into the category of $\mathrm{C}^{*}$-correspondences over the corresponding fixed point algebra (cf. [17, Section 2]). More detailedly, the paper is organized as follows.

After some preliminaries, we introduce in Section 3 the notion of freeness for compact $\mathrm{C}^{*}$ dynamical systems and prove its equivalence to the Ellwood condition (Theorem 3.2). We also list a few examples and establish the basis for our later classification in terms of generalized factor systems. In Section 4 we show that every free compact $\mathrm{C}^{*}$-dynamical system gives rise to a so-called factor system and that free compact $\mathrm{C}^{*}$-dynamical systems can be classified up to equivalence by their associated factor system (Theorem 4.4). This extends the results presented in part 2 of this series [28], which deals with the particular class of cleft actions. Moreover, we give a characterization of cleft actions in terms of their factor systems. The purpose of Section 5 is to show that the information provided by a factor system is enough to explicitly reconstruct the $\mathrm{C}^{*}$-dynamical system by adapting results of [17]. This completes our classification result showing that there is a 1-to- 1 correspondence between free compact $\mathrm{C}^{*}$-dynamical systems and factor systems up to equivalence and conjugacy, respectively (Theorem 5.6). As an application, we show in Section 6 that finite coverings of generic irrational rotation $\mathrm{C}^{*}$-algebras are always cleft (Theorem 6.4).

\section{Preliminaries and notations}

Our study is concerned with free actions of compact groups on unital $\mathrm{C}^{*}$-algebras and their classification in terms of generalized factor systems. Consequently, we use and blend tools from operator algebras and representation theory. In this preliminary section we provide definitions and notations which are repeatedly used in this article.

\section{$\mathrm{C}^{*}$-algebras}

Let $\mathcal{A}$ be a unital $\mathrm{C}^{*}$-algebra. For the unit of $\mathcal{A}$ we write $\mathbb{1}_{\mathcal{A}}$ or simply $\mathbb{1}$. We will frequently deal with partial isometries, i.e., elements $v \in \mathcal{A}$ such that $v^{*} v$ and $v v^{*}$ are projections. In this case $v^{*} v$ is called the cokernel projection and $v v^{*}$ the range projection. Moreover, we say that a projection $p$ is larger than the range of an element $x$ if $p x=x$, and we say that $p$ is larger than the cokernel of $x$ if $x p=x$. All tensor products of $\mathrm{C}^{*}$-algebras are taken with respect to 
the minimal tensor product. We will frequently deal with multiple tensor products of unital $\mathrm{C}^{*}$-algebras $\mathcal{A}, \mathcal{B}$, and $\mathcal{C}$. If there is no ambiguity, we regard $\mathcal{A}, \mathcal{B}$, and $\mathcal{C}$ as subalgebras of $\mathcal{A} \otimes \mathcal{B} \otimes \mathcal{C}$ and extend maps on $\mathcal{A}, \mathcal{B}$, or $\mathcal{C}$ canonically by tensoring with the identity map. For sake of clarity we may occasionally use the leg numbering notation, e.g., for $x \in \mathcal{A} \otimes \mathcal{C}$ we write $x_{13}$ to denote the corresponding element in $\mathcal{A} \otimes \mathcal{B} \otimes \mathcal{C}$.

Inner products $\langle\cdot, \cdot\rangle$ on a Hilbert space is always assumed to be linear in the second component. For a Hilbert space $\mathfrak{H}_{1}, \mathfrak{H}_{2}$ we denote by $\mathcal{L}\left(\mathfrak{H}_{1}, \mathfrak{H}_{2}\right)$ the set of bounded linear operators $T: \mathfrak{H}_{1} \rightarrow \mathfrak{H}_{2}$. If $\mathfrak{H}_{1}=\mathfrak{H}_{2}$ we briefly write $\mathcal{L}\left(\mathfrak{H}_{1}\right)$. We use the Dirac notation to specify operators, i.e., for two vectors $v_{1} \in \mathfrak{H}_{1}, v_{2} \in \mathfrak{H}_{2}$ we write $\left|v_{2}\right\rangle\left\langle v_{1}\right|$ for the operator $v \mapsto\left\langle v_{1}, v\right\rangle v_{2}$.

\section{Hilbert modules}

For a unital $\mathrm{C}^{*}$-algebra $\mathcal{A}$ a right pre-Hilbert $\mathcal{A}$-module is a right $\mathcal{A}$-module $\mathfrak{H}$ equipped with a sesquilinear map $\langle\cdot, \cdot\rangle_{\mathcal{A}}: \mathfrak{H} \times \mathfrak{H} \rightarrow \mathcal{A}$ that satisfies the usual axioms of a definite inner product with $\mathcal{A}$-linearity in the second component. We call $\mathfrak{H}$ a right Hilbert $\mathcal{A}$-module if $\mathfrak{H}$ is complete with respect to the norm $\|x\|_{\mathfrak{H}}:=\left\|\langle x, x\rangle_{\mathcal{A}}\right\|^{1 / 2}$. The right Hilbert $\mathcal{A}$-module is called full if the two-sided ideal $\langle\mathfrak{H}, \mathfrak{H}\rangle_{\mathcal{A}}:=\overline{\operatorname{lin}\left\{\langle x, y\rangle_{\mathcal{A}} \mid x, y \in \mathfrak{H}\right\}}$ is dense in $\mathcal{A}$. Since every dense ideal of $\mathcal{A}$ meets the invertible elements, in this case we have $\langle\mathfrak{H}, \mathfrak{H}\rangle_{\mathcal{A}}=\mathcal{A}$. Left (pre-) Hilbert $\mathcal{A}$-modules are defined in a similar way.

A correspondence over $\mathcal{A}$, or a right Hilbert $\mathcal{A}$-bimodule, is a $\mathcal{A}$-bimodule $\mathfrak{H}$ equipped with a $\mathcal{A}$-valued inner product $\langle\cdot, \cdot\rangle_{\mathcal{A}}$ which turns it into a right Hilbert $\mathcal{A}$-module such that the left action of $\mathcal{A}$ on $\mathfrak{H}$ is via adjointable operators. For two correspondences $\mathfrak{H}$ and $\mathfrak{K}$ over $\mathcal{A}$ we denote by $\mathfrak{H} \otimes_{\mathcal{A}} \mathfrak{K}$ their tensor product, on which the inner product is given by $\left\langle x_{1} \otimes y_{1}, x_{2} \otimes y_{2}\right\rangle_{\mathcal{A}}=$ $\left\langle y_{1},\left\langle x_{1}, x_{2}\right\rangle_{\mathcal{A}} \cdot y_{2}\right\rangle_{\mathcal{A}}$ for all $x_{1}, x_{2} \in \mathfrak{H}$ and $y_{1}, y_{2} \in \mathfrak{K}$.

\section{Compact quantum groups}

We rely on the $\mathrm{C}^{*}$-algebraic notion of compact quantum groups as introduced by Woronowicz [34]. For an introduction and further details we recommend [6, 18, 29]. A compact quantum group is given by a unital $\mathrm{C}^{*}$-algebra $\mathcal{G}$ together with a (usually implicit) faithful, unital *-homomorphism $\Delta: \mathcal{G} \rightarrow \mathcal{G} \otimes \mathcal{G}$ satisfying the identity $(\Delta \otimes$ id $) \circ \Delta=($ id $\otimes \Delta) \circ \Delta$ and such that $\Delta(\mathcal{G})(\mathbb{1} \otimes \mathcal{G})$ is dense in $\mathcal{G} \otimes \mathcal{G}$. It can be shown that there is a unique state $h: \mathcal{G} \rightarrow \mathbb{C}$ such that (id $\otimes h) \circ \Delta=h=(h \otimes \mathrm{id}) \circ \Delta$ (see [34]). This state is called the Haar state of $\mathcal{G}$. It is not faithful in general but via the GNS-construction we may replace $\mathcal{G}$ by its reduced version on which the Haar state is faithful. Since $\mathcal{G}$ and its reduce version behave identically with respect to their representation theory and their actions (see [6, Section 4]), we will throughout the text assume that the Haar state on $\mathcal{G}$ is faithful.

A unitary representation of a compact quantum group $\mathcal{G}$ on a finite-dimensional Hilbert space $V$ is a unitary element $\pi \in \mathcal{L}(V) \otimes \mathcal{G}$ such that id $\otimes \Delta(\pi)=\pi_{12} \pi_{13}$ in $\mathcal{L}(V) \otimes \mathcal{G} \otimes \mathcal{G}$. Unless explicitly stated otherwise, all representations are assumed unitary and finite-dimensional. We recall that the set of equivalence classes of irreducible representations $\hat{\mathcal{G}}$ is countable and that the matrix coefficients of all $\pi \in \hat{\mathcal{G}}$ generate a dense ${ }^{*}$-subalgebra of $\hat{\mathcal{G}}$. Since all constructions behave naturally with respect to intertwiners we will not distinguish between a representation and its equivalence class. The tensor product of two representations $(\pi, V)$ and $(\rho, W)$ of $\mathcal{G}$ is the representation $(\pi \otimes \rho, V \otimes W)$ given by the unitary element $\pi \otimes \rho:=\pi_{13} \rho_{23}$ in $\mathcal{L}(V) \otimes \mathcal{L}(W) \otimes \mathcal{G}$. We also recall that for a representation $(\pi, V)$ of $\mathcal{G}$ the contragradient representation is in general not unitary. Its normalization $(\bar{\pi}, \bar{V})$ is called the conjugated representation.

Some care has to be taken in the case that the Haar state is not tracial. Then the matrix coefficients with respect to some chosen basis of $V$ are not orthogonal in general. However, if $\pi$ is irreducible, there is a unique positive, invertible operator $Q(\pi) \in \mathcal{L}(V)$ normalized to 
$\operatorname{Tr}[Q(\pi)]=\operatorname{Tr}\left[Q(\pi)^{-1}\right]$ with

$$
\frac{\operatorname{Tr}[Q(\pi) T]}{\operatorname{Tr}[Q(\pi)]} \mathbb{1}_{V}=\mathrm{id} \otimes h\left(\pi\left(T \otimes \mathbb{1}_{\mathcal{G}}\right) \pi^{*}\right), \quad \frac{\operatorname{Tr}\left[Q(\pi)^{-1} T\right]}{\operatorname{Tr}[Q(\pi)]} \mathbb{1}_{V}=\mathrm{id} \otimes h\left(\pi^{*}\left(T \otimes \mathbb{1}_{\mathcal{G}}\right) \pi\right)
$$

for every $T \in \mathcal{L}(V)$. The number $d_{\pi}:=\operatorname{Tr}[Q(\pi)]$ is called the quantum dimension of $\pi$. The quantum dimension behaves nicely with respect to taking direct sums, tensor products, and conjugated representations. An important detail for us is the fact that we may fix intertwiners $R: \mathbb{C} \rightarrow V \otimes \bar{V}$ and $\bar{R}: \mathbb{C} \rightarrow \bar{V} \otimes V$ for all irreducible representation such that $\left(R^{*} \otimes \mathrm{id}_{V}\right)\left(\mathrm{id}_{V} \otimes \bar{R}\right)=\mathrm{id}_{V}$. In terms of an orthonormal basis $e_{1}, \ldots, e_{n} \in V$ and its respective conjugated basis $\bar{e}_{1}, \ldots, \bar{e}_{n} \in \bar{V}$ we typically choose

$$
R(1)=\sum_{i=1}^{n} Q(\pi)^{1 / 2} e_{i} \otimes \bar{e}_{i} .
$$

\section{Actions of compact quantum groups}

An action of a compact quantum group $\mathcal{G}$ on a unital $\mathrm{C}^{*}$-algebra $\mathcal{A}$ is a faithful, unital *-homomorphism $\alpha: \mathcal{A} \rightarrow \mathcal{A} \otimes \mathcal{G}$ that satisfies $(\mathrm{id} \otimes \Delta) \circ \alpha=(\alpha \otimes \mathrm{id}) \circ \alpha$ and such that $(\mathbb{1} \otimes \mathcal{G}) \alpha(\mathcal{A})$ is dense in $\mathcal{A} \otimes \mathcal{G}$. Since we assume that the Haar state is faithful, the map $P_{1}:=(\mathrm{id} \otimes h) \circ \alpha$ is a faithful conditional expectation onto the fixed point algebra

$$
\mathcal{A}^{\mathcal{G}}:=\left\{x \in \mathcal{A} \mid \alpha(x)=x \otimes \mathbb{1}_{\mathcal{G}}\right\} .
$$

In particular, $\mathcal{A}$ turns into a right pre-Hilbert $\mathcal{A}^{\mathcal{G}}$-bimodule with the $\mathcal{A}^{\mathcal{G}}$-valued inner product $\langle x, y\rangle_{\mathcal{A}^{\mathcal{G}}}:=P_{1}\left(x^{*} y\right)$ for $x, y \in \mathcal{A}$. For each irreducible representation $\pi \in \hat{G}$ the projection $P_{\pi}: \mathcal{A} \rightarrow \mathcal{A}$ onto the $\pi$-isotypic component $A(\pi):=P_{\pi}(\mathcal{A})$ is given by

$$
P_{\pi}(a):=d_{\pi} \operatorname{Tr} \otimes \operatorname{id}_{\mathcal{A}} \otimes h\left(\bar{\pi}_{13} \alpha(a)_{23} Q(\bar{\pi})_{1}^{-1}\right), \quad a \in \mathcal{A},
$$

where the leg numbering refers to $\mathcal{L}(\bar{V}) \otimes \mathcal{A} \otimes \mathcal{G}$ (see [23, Theorem 1.5]). The set $A(\pi)$ is in fact closed with respect to the inner product (see [7, Corollary 2.6]) and hence a correspondence over $\mathcal{A}^{\mathcal{G}}$. Furthermore, isotypic components for different $\pi \in \hat{\mathcal{G}}$ are orthogonal with respect to the inner product and the sum $\sum_{\pi \in \hat{\mathcal{G}}} A(\pi)$ is dense in $\mathcal{A}$.

\section{$3 \quad$ Free $\mathrm{C}^{*}$-dynamical systems}

Throughout the presentation we discuss compact $\mathrm{C}^{*}$-dynamical systems $(\mathcal{A}, \mathcal{G}, \alpha)$, by which we mean a unital $\mathrm{C}^{*}$-algebra $\mathcal{A}$, a compact quantum group $\mathcal{G}$, and an action $\alpha: \mathcal{A} \rightarrow \mathcal{A} \otimes \mathcal{G}$. Given such a system, we recall that $\mathcal{A}$ can be decomposed in terms of its isotypic components $A(\pi)$, $\pi \in \hat{\mathcal{G}}$, and that each $A(\pi)$ is a correspondence over the fixed point algebra $\mathcal{A}^{\mathcal{G}}$. For each irreducible representation $(\pi, V) \in \hat{\mathcal{G}}$ we denote by $\Gamma(V)$ the multiplicity space of the conjugated representation $\bar{\pi}$, which can be written in the form

$$
\Gamma(V)=\left\{x \in V \otimes \mathcal{A} \mid \pi_{13} \mathrm{id}_{V} \otimes \alpha(x)=x \otimes \mathbb{1}_{\mathcal{G}}\right\} .
$$

This space is naturally a correspondence over $\mathcal{A}^{\mathcal{G}}$ with respect to the usual left and right multiplication and the restriction of the inner product $\langle v \otimes a, w \otimes b\rangle_{\mathcal{A}^{\mathcal{G}}}:=\langle v, w\rangle a^{*} b$ for all $v, w \in V$ and $a, b \in \mathcal{A}$. The $\pi$-isotypic component $A(\pi)$ is then as a correspondence isomorphic to $V \otimes \Gamma(\bar{V})$ via the map $\varphi_{\pi}: V \otimes \Gamma(\bar{V}) \rightarrow A(\pi), \varphi_{\pi}:=d_{\pi}^{-1 / 2} R^{*} \otimes \operatorname{id}_{\mathcal{A}}$.

The mapping $(\pi, V) \mapsto \Gamma(V)$ can be extended to an additive functor from the representation category of $\mathcal{G}$ into the category of $\mathrm{C}^{*}$ - correspondences over $\mathcal{A}^{\mathcal{G}}$. Since $\mathcal{A}$ is the closure of 
the direct sum of its isotypic components and every isotypic component $A(\pi)$ is isomorphic to $V \otimes \Gamma(\bar{V})$, this functor allows us to reconstruct the Hilbert $\mathcal{A}^{\mathcal{G}}$-bimodule structure of $\mathcal{A}$ and the action $\alpha$ up to a suitable closure. To recover the multiplication on $\mathcal{A}$ we may look at the family of maps

$$
m_{\pi, \rho}: \quad \Gamma(V) \otimes_{\mathcal{B}} \Gamma(W) \rightarrow \Gamma(V \otimes W), \quad m_{\pi, \rho}(x \otimes y):=x_{13} y_{23},
$$

for representations $(\pi, V),(\rho, W)$ of $\mathcal{G}$. Here the subindices on the right hand side refer to the leg numbering in $V \otimes W \otimes \mathcal{A}$, that is, for elementary tensors $x=(v \otimes a)$ and $y=(w \otimes b)$ we write $x_{13} y_{23}=v \otimes w \otimes a b(v \in V, w \in W, a, b \in \mathcal{A})$. The functor $\Gamma: V \mapsto \Gamma(V)$ and the transformations $\left(m_{\pi, \rho}\right)_{\pi, \rho}$ constitute a so-called weak tensor functor and allow to recover the reduced form of the compact $\mathrm{C}^{*}$-dynamical system $(\mathcal{A}, \mathcal{G}, \alpha)$ up to isomorphisms (see [17, Section 2]).

To obtain a more concrete representation we restrict ourselves to the class of free action in the following sense. In addition to the above correspondence structure, we equip each multiplicity space $\Gamma(V)$ with the left $\mathcal{L}(V) \otimes \mathcal{A}$-valued inner product given by

$$
\mathcal{L}(V) \otimes \mathcal{A}\langle v \otimes a, w \otimes b\rangle:=|v\rangle\langle w| \otimes a b^{*}
$$

for $v, w \in V$ and $a, b \in \mathcal{A}$. A few moments thought show that this left inner product takes values in the $\mathrm{C}^{*}$-algebra $\left\{x \in \mathcal{L}(V) \otimes \mathcal{A} \mid \alpha(x)=\pi\left(x \otimes \mathbb{1}_{\mathcal{G}}\right) \pi^{*}\right\}$ and that the only missing feature for $\Gamma(V)$ to be a Morita equivalence bimodule is that in general the left inner product need not be full. This requirement is what we demand for a free action:

Definition 3.1. A compact $\mathrm{C}^{*}$-dynamical system $(\mathcal{A}, \mathcal{G}, \alpha)$ is called free if for every $(\pi, V) \in \hat{\mathcal{G}}$ we have $\mathbb{1} \in \mathcal{L}(V) \otimes \mathcal{A}\langle\Gamma(V), \Gamma(V)\rangle$.

There are various non-equivalent notions of freeness in the literature (see, e.g., [9, 22] and references therein). The one given here was introduced for actions of classical compact groups by Rieffel [26] under the term saturated actions (see also [20, Corollary 3.5] and [13, Lemma 3.1]) and already used in the other parts of this series [27, 28], where some equivalent conditions are summarized. A seemingly different version of freeness for actions of compact quantum groups was recently exploited by De Commer et al. [3, 7] and is due to D.A. Ellwood [10]. We recall that a compact $\mathrm{C}^{*}$-dynamical system $(\mathcal{A}, \mathcal{G}, \alpha)$ is said to satisfy the Ellwood condition if $(\mathcal{A} \otimes \mathbb{1}) \alpha(\mathcal{A})$ is dense in $\mathcal{A} \otimes \mathcal{G}$. For convenience we now summarize the equivalent conditions of freeness and provide proper references for the implications.

Theorem 3.2. Let $(\mathcal{A}, \mathcal{G}, \alpha)$ be a compact $\mathrm{C}^{*}$-dynamical system. Then the following conditions are equivalent:

(a) The $\mathrm{C}^{*}$-dynamical system $(\mathcal{A}, \mathcal{G}, \alpha)$ is free.

(b) For all representations $(\pi, V),(\rho, W)$ of $\mathcal{G}$ the map $m_{\pi, \rho}$ defined in equation (3.1) has dense range or, equivalently, is surjective.

(c) The $\mathrm{C}^{*}$-dynamical system $(\mathcal{A}, G, \alpha)$ satisfies the Ellwood condition.

The equivalence between (b) and (c) was proved quite recently in [3, Theorem 0.4]. For the implication (c) $\Rightarrow$ (a) we refer to the proof of [7, Corollary 5.6]. Finally, the implication (a) $\Rightarrow$ (b) will follow immediately from the independent later results of Section 4 and from Lemma 5.3. Alternatively, (b) follows from (a) by observing that an equivalent way to formulate the condition in Definition 3.1 is by saying that, for every representation $(\pi, V)$ of $\mathcal{G}$, the right Hilbert $\mathcal{A}$-module $V \otimes \mathcal{A}$ has a basis (in the sense of Hilbert modules) consisting of invariant elements.

We continue with a reformulation of freeness which will be convenient for our description of free $\mathrm{C}^{*}$-dynamical systems in terms of generalized factor systems. 
Lemma 3.3. A compact $\mathrm{C}^{*}$-dynamical system $(\mathcal{A}, \mathcal{G}, \alpha)$ is free if and only if for every representation $(\pi, V)$ of $\mathcal{G}$ there is a finite-dimensional Hilbert space $\mathfrak{H}$ and a coisometry $s \in \mathcal{L}(\mathfrak{H}, V) \otimes \mathcal{A}$ with $\pi_{13}$ id $\otimes \alpha(s)=s \otimes \mathbb{1}_{\mathcal{G}}$.

Proof. For the "if"-implication let $(\pi, V) \in \hat{\mathcal{G}}$ and let $s \in \mathcal{L}(\mathfrak{H}, V) \otimes \mathcal{A}$ be a coisometry with $\pi \alpha(s)=s$. Moreover, fix an orthonormal basis of $\mathfrak{H}$ and denote by $s_{k} \in V \otimes \mathcal{A}$ the columns of $s$. Then

$$
\sum_{k=1}^{n} \mathcal{L}(V) \otimes \mathcal{A}\left\langle s_{k}, s_{k}\right\rangle=s s^{*}=\mathbb{1} .
$$

For the converse implication, first observe that freeness of the $\mathrm{C}^{*}$-dynamical system $(\mathcal{A}, \mathcal{G}, \alpha)$ implies that, for each representation $(\pi, V)$ of $\mathcal{G}$, the space $\Gamma(V)$ is a Morita equivalence bimodule between the $\mathrm{C}^{*}$-algebras $C(\pi):=\left\{x \in \mathcal{L}(V) \otimes \mathcal{A} \mid \mathrm{id} \otimes \alpha(x)=\pi_{13}\left(x \otimes \mathbb{1}_{\mathcal{G}}\right) \pi_{13}^{*}\right\}$ and $\mathcal{A}^{\mathcal{G}}$. Since $C(\pi)$ is unital, there are elements $s_{1}, \ldots, s_{n} \in \Gamma(V)$ such that

$$
\sum_{k=1}^{n} \mathcal{L}(V) \otimes \mathcal{A}\left\langle s_{k}, s_{k}\right\rangle=\mathbb{1}
$$

(see Lemma A.1). Now put $\mathfrak{H}:=\mathbb{C}^{n}$ and denote by $s \in \mathcal{L}(\mathfrak{H}, V) \otimes \mathcal{A}$ the element with columns $s_{1}, \ldots, s_{n}$ in the canonical orthonormal basis. Then $\pi \alpha(s)=s \otimes \mathbb{1}_{\mathcal{G}}$, since $s_{k} \in \Gamma(V)$, and further

$$
s s^{*}=\sum_{k=1}^{n} \mathcal{L}(V) \otimes \mathcal{A}\left\langle s_{k}, s_{k}\right\rangle=\mathbb{1} .
$$

Remark 3.4. For each representation $\pi$ of $\mathcal{G}$ there is a minimal dimension, say $n(\pi)$, that the Hilbert space $\mathfrak{H}$ in Lemma 3.3 can take. Clearly we have $n(1)=1, n(\pi \oplus \rho) \leq n(\pi)+n(\rho)$, and $n(\pi \otimes \rho) \leq n(\pi) \cdot n(\rho)$, using a variant of the multiplication map $m_{\pi, \rho}$.

Suppose we fix a Hilbert space $\mathfrak{H}_{\pi}$ and a respective coisometry $s(\pi)$ for each irreducible representation $\pi \in \hat{\mathcal{G}}$. Then we may extend $\pi \mapsto \mathfrak{H}_{\pi}$ to an additive functor and $\pi \mapsto s(\pi)$ to a family of coisometries that satisfies the condition in Lemma 3.3 and behaves naturally with respect to intertwiners. However, the functor $\pi \mapsto \mathfrak{H}_{\pi}$ is in general not a tensor functor and $s(\pi \otimes \rho)$ has no immediate relation to $s(\pi)$ and $s(\rho)$.

In the remaining part of this section we present a bouquet of examples. To begin with, we recall that Definition 3.1 actually extends the classical notion of free actions of compact groups. In fact, given a compact space $P$ and a compact group $G$, it is a consequence of [21, Proposition 7.1.12 and Theorem 7.2.6] that a continuous group action $\sigma: P \times G \rightarrow P$ is free, i.e., its stabilizer groups vanish at each point, if and only if the induced $\mathrm{C}^{*}$-dynamical system $\left(C(P), G, \alpha_{\sigma}\right)$ is free in the sense of Definition 3.1. Therefore, Definition 3.1 also provides a natural framework for noncommutative principal bundles. Furthermore, we would like to point out that Definition 3.1 characterizes classical free group actions in terms of associated vector bundles and the condition therein means that the associated vector bundles have non-degenerate fibres (see, e.g., [30]).

Example 3.5. We would like to recall a $\mathrm{C}^{*}$-algebraic version of the nontrivial Hopf-Galois extension studied in [15] (see also [5]). Let $\theta \in \mathbb{R}$ be fixed and let $\theta^{\prime}$ be the skewsymmetric $4 \times 4$-matrix with $\theta_{1,2}^{\prime}=\theta_{3,4}^{\prime}=0$ and $\theta_{1,3}^{\prime}=\theta_{1,4}^{\prime}=\theta_{2,3}^{\prime}=\theta_{2,4}^{\prime}=\theta / 2$. We consider the universal unital $\mathrm{C}^{*}$-algebra $\mathcal{A}\left(\mathbb{S}_{\theta^{\prime}}^{7}\right)$ generated by normal elements $z_{1}, \ldots, z_{4}$ satisfying the relations

$$
z_{i} z_{j}=e^{2 \pi \imath \theta_{i, j}^{\prime}} z_{j} z_{i}, \quad z_{j}^{*} z_{i}=e^{2 \pi \imath \theta_{i, j}^{\prime}} z_{i} z_{j}^{*}, \quad \sum_{k=1}^{4} z_{k}^{*} z_{k}=\mathbb{1}
$$


for all $1 \leq i, j \leq 4$. A few moments thought show that the group $G=\mathrm{SU}(2)$ acts strongly continuously on $\mathcal{A}\left(\mathbb{S}_{\theta^{\prime}}^{7}\right)$ via the ${ }^{*}$-automorphisms $\left(\alpha_{U}\right)_{U \in \mathrm{SU}(2)}$ given on generators by

$$
\alpha_{U}:\left(z_{1}, \ldots, z_{4}\right) \mapsto\left(z_{1}, \ldots, z_{4}\right)\left(\begin{array}{cc}
U & 0 \\
0 & U
\end{array}\right)
$$

Moreover, the fixed point algebra turns out to be the universal unital $\mathrm{C}^{*}$-algebra $\mathcal{A}\left(\mathbb{S}_{\theta}^{4}\right)$ generated by normal elements $w_{1}, w_{2}$ and a self-adjoint element $x$ satisfying

$$
w_{1} w_{2}=e^{2 \pi \imath \theta} w_{2} w_{1}, \quad w_{2}^{*} w_{1}=e^{2 \pi \imath \theta} w_{1} w_{2}^{*}, \quad \text { and } \quad w_{1}^{*} w_{1}+w_{2}^{*} w_{2}+x^{*} x=\mathbb{1} .
$$

For $\theta=0$ all algebras are commutative and we recover the classical 7-dimensional Hopf fibration of the 4-sphere, which is a well-known example of a non-trivial principal bundle. Many arguments from the classical case can be extended to arbitrary $\theta$. In particular, it is easily checked that for the fundamental 2-dimensional representation $\left(\pi_{1}, \mathbb{C}^{2}\right)$ of $\mathrm{SU}(2)$ a coisometry $s \in \mathcal{L}\left(\mathbb{C}^{4}, \mathbb{C}^{2}\right) \otimes \mathcal{A}\left(\mathbb{S}_{\theta^{\prime}}^{7}\right)$ with $U \alpha_{U}(s)=s$ for all $U \in \mathrm{SU}(2)$ is given by

$$
s:=\left(\begin{array}{cccc}
z_{1}^{*} & -z_{2} & z_{3}^{*} & -z_{4} \\
z_{2}^{*} & z_{1} & z_{4}^{*} & z_{3}
\end{array}\right) .
$$

Since every irreducible representation of $\mathrm{SU}(2)$ can be obtained as a subrepresentation of a suitable tensor powers of $\pi_{1}$, we may take tensor products of $s$ with itself in order to find a suitable coisometry for every representation $\pi$ of $\mathrm{SU}(2)$. We conclude that the compact $\mathrm{C}^{*}$-dynamical system $\left(\mathcal{A}\left(\mathbb{S}_{\theta^{\prime}}^{7}\right), \mathrm{SU}(2), \alpha\right)$ is free.

\section{Example 3.6.}

1. Bichon, De Rijdt and Vaes introduce in [4] the notion of quantum multiplicity of an irreducible representation in an ergodic action of a compact quantum group and classify ergodic actions of so-called full quantum multiplicity in terms of unitary fiber functors. It follows from [4, Theorem 3.9] that these actions are free.

2. According to [4, Corollary 5.8], for sufficiently small parameters $q$ the compact quantum group $\mathrm{SU}_{q}(2)$ admits an ergodic action of full quantum multiplicity such that the multiplicity of the fundamental representation is arbitrarily large. Hence, there are plenty of free and ergodic actions of $\mathrm{SU}_{q}(2)$.

Example 3.7. Let $\mathcal{G}$ be an $R^{+}$-deformation (see, e.g., [2, Theorem 2.1]) of a semisimple compact Lie group. Furthermore, let $\left(\pi, \mathbb{C}^{d}\right)$ be a faithful representation of $\mathcal{G}$. Then [11, Proposition 7.3] implies that the induced action $\alpha$ of $\mathcal{G}$ on the Cuntz algebra $\mathcal{O}_{d}$ defined by

$$
\alpha\left(S_{i}\right):=\sum_{j=1}^{d} S_{j} \otimes \pi_{j, i}
$$

is free, where $S_{1}, \ldots S_{d}$ denote the generators of $\mathcal{O}_{d}$. It is not hard to check that for the representation $\left(\pi, \mathbb{C}^{d}\right)$ a coisometry $s \in \mathcal{L}\left(\mathbb{C}, \mathbb{C}^{d}\right) \otimes \mathcal{O}_{d}$ with $\pi_{13}$ id $\otimes \alpha(s)=s \otimes \mathbb{1}_{\mathcal{G}}$ is given by

$$
s:=\left(S_{1}^{*}, S_{2}^{*}, \ldots, S_{d}^{*}\right)^{\top} .
$$

\section{Factor systems}

We have seen in Lemma 3.3 that freeness of a compact $\mathrm{C}^{*}$-dynamical system $(\mathcal{A}, \mathcal{G}, \alpha)$ can be cast in form of a family of coisometries. These coisometries may be used to give a more explicit picture 
of the spectral subspaces of the $\mathrm{C}^{*}$-dynamical system. In fact, let $(\pi, V)$ be a representation of $\mathcal{G}$ and let $s(\pi) \in \mathcal{L}(\mathfrak{H}, V) \otimes \mathcal{A}$ be a coisometry with $\pi_{13}$ id $\otimes \alpha(s(\pi))=s(\pi) \otimes \mathbb{1}_{\mathcal{G}}$ in $\mathcal{L}(\mathfrak{H}, V) \otimes \mathcal{A} \otimes \mathcal{G}$. Then a few moments thought show that the multiplicity space $\Gamma(V) \subseteq V \otimes \mathcal{A}$ is the range of the element $s(\pi)$, i.e., we have

$$
\Gamma(V)=s(\pi)\left(\mathfrak{H} \otimes \mathcal{A}^{\mathcal{G}}\right)
$$

The explicit form allows us to phrase the correspondence structure and the multiplicative structure among the generalized isotypic components only in terms of the fixed point algebra $\mathcal{A}^{G}$ and the quantum group $\mathcal{G}$. This fact was already exploited in the previous part of this series [28], where we carried out the analysis in the case of cleft dynamical systems with a classical compact group. With some adjustments we generalize the construction here to arbitrary free $\mathrm{C}^{*}$-dynamical systems and quantum groups.

We start with a free compact $\mathrm{C}^{*}$-dynamical system $(\mathcal{A}, \mathcal{G}, \alpha)$ and we write briefly $\mathcal{B}:=\mathcal{A}^{\mathcal{G}}$ for the corresponding fixed point algebra. Furthermore, we choose a functorial version of the finite-dimensional Hilbert spaces $\mathfrak{H}_{\pi}$ and the coisometries $s(\pi)$ for each representation $\pi$ of $\mathcal{G}$ (see also the discussion after Lemma 3.3). In particular, we assume without loss of generality that $\mathfrak{H}_{1}=\mathbb{C}$ and $s(1)=\mathbb{1}_{\mathcal{B}}$. Then we consider for each representation $\pi$ of $\mathcal{G}$ the ${ }^{*}$-homomorphism

$$
\gamma_{\pi}: \mathcal{B} \rightarrow \mathcal{L}\left(\mathfrak{H}_{\pi}\right) \otimes \mathcal{B}, \quad \gamma_{\pi}(b):=s(\pi)^{*}\left(\mathbb{1}_{V_{\pi}} \otimes b\right) s(\pi)
$$

and for each pair $\pi, \rho$ of representations of $\mathcal{G}$ the element

$$
\omega(\pi, \rho):=s(\pi \otimes \rho)^{*} s(\pi) s(\rho) \in \mathcal{L}\left(\mathfrak{H}_{\pi} \otimes \mathfrak{H}_{\rho}, \mathfrak{H}_{\pi \otimes \rho}\right) \otimes \mathcal{B},
$$

where $s(\pi)$ and $s(\rho)$ are amplified to act trivially on $\mathfrak{H}_{\rho}$ and $\mathfrak{H}_{\pi}$, respectively.

Definition 4.1. Let $(\mathcal{A}, \mathcal{G}, \alpha)$ be a free compact $\mathrm{C}^{*}$-dynamical system. Then the system $(\mathfrak{H}, \gamma, \omega)=\left(\mathfrak{H}_{\pi}, \gamma_{\pi}, \omega(\pi, \rho)\right)_{\pi, \rho \in \hat{\mathcal{G}}}$ constructed above is called a factor system of $(\mathcal{A}, \mathcal{G}, \alpha)$.

Remark 4.2. For some computations it is convenient to express the factor system in terms of fixed orthonormal bases of the Hilbert spaces $\mathfrak{H}_{\pi}, \pi \in \hat{\mathcal{G}}$. In this situation we denote by $s(\pi)_{1}, \ldots, s(\pi)_{n} \in \Gamma(V)$ the columns of $s(\pi)$. Then the ${ }^{*}$-homomorphism $\gamma_{\pi}: \mathcal{B} \rightarrow M_{n} \otimes \mathcal{B}$ has the coefficients

$$
\gamma_{\pi}(b)_{i, j}=\left\langle s(\pi)_{i}, b . s(\pi)_{j}\right\rangle_{\mathcal{B}}
$$

for all $1 \leq i \leq \operatorname{dim} \mathfrak{H}_{\pi}$ and $1 \leq j \leq \operatorname{dim} \mathfrak{H}_{\rho}$. For the partial isometry $\omega(\pi, \rho)$ we first fix an irreducible subrepresentation $\sigma$ of $\pi \otimes \rho$. Then the coefficients on the corresponding subspace $\mathfrak{H}_{\sigma} \subseteq \mathfrak{H}_{\pi} \otimes \mathfrak{H}_{\rho}$ are given by

$$
\omega(\pi, \rho)_{(i, j), k}=\left\langle m\left(s(\pi)_{i} \otimes s(\rho)_{j}, s(\sigma)_{k}\right\rangle_{\mathcal{B}}\right.
$$

for all $1 \leq i \leq \operatorname{dim} \mathfrak{H}_{\pi}, 1 \leq j \leq \operatorname{dim} \mathfrak{H}_{\rho}$, and $1 \leq k \leq \operatorname{dim} \mathfrak{H}_{\sigma}$.

Of course, different choices of Hilbert spaces $\mathfrak{H}_{\pi}$ and coisometries $s(\pi)$ give rise to different factor systems. However, as the following lemma shows, those choices only effect the factor system by a conjugacy with partial isometries:

Lemma 4.3 (cf. Lemma 5.5 and Theorem 5.6 in [28]). For a factor system $(\mathfrak{H}, \gamma, \omega)$ of a free compact $\mathrm{C}^{*}$-dynamical system $(\mathcal{A}, \mathcal{G}, \alpha)$ with fixed point algebra $\mathcal{B}$ the following assertions hold: 
1. We have $\omega(1,1)=\mathbb{1}_{\mathcal{B}}, \gamma_{1}=\operatorname{id}_{\mathcal{B}}$ and

$$
\begin{aligned}
& \omega(\pi, \rho) \omega(\pi, \rho)^{*}=\gamma_{\pi \otimes \rho}(\mathbb{1}), \quad \omega(\pi, \rho)^{*} \omega(\pi, \rho)=\mathrm{id} \otimes \gamma_{\rho}\left(\gamma_{\pi}(\mathbb{1})\right), \\
& \gamma_{\pi \otimes \rho}(b) \omega(\pi, \rho)=\omega(\pi, \rho) \gamma_{\rho}\left(\gamma_{\pi}(b)\right), \\
& \omega(\pi, \rho \otimes \sigma)(\mathbb{1} \otimes \omega(\rho, \sigma))=\omega(\pi \otimes \rho, \sigma) \mathrm{id} \otimes \gamma_{\sigma}(\omega(\pi, \rho))
\end{aligned}
$$

for all representations $\pi, \rho$ of $\mathcal{G}$ and $b \in \mathcal{B}$. We refer to the equation (4.2) as the coaction condition and to equation (4.3) as the cocycle condition.

2. Let $\left(\mathfrak{H}^{\prime}, \gamma^{\prime}, \omega^{\prime}\right)$ be another factor system of $(\mathcal{A}, \mathcal{G}, \alpha)$. Then there is a family of partial isometries $v(\pi) \in \mathcal{L}\left(\mathfrak{H}_{\pi}^{\prime}, \mathfrak{H}_{\pi}\right) \otimes \mathcal{B}, \pi \in \hat{\mathcal{G}}$, such that

$$
\begin{aligned}
& v(\pi) v(\pi)^{*}=\gamma_{\pi}(\mathbb{1}), \quad v(\pi)^{*} v(\pi)=\gamma_{\pi}^{\prime}(\mathbb{1}), \\
& v(\pi) \gamma_{\pi}^{\prime}(b)=\gamma_{\pi}(b) v(\pi), \\
& v(\pi \otimes \rho) \omega^{\prime}(\pi, \rho)=\omega(\pi, \rho) \text { id } \otimes \gamma_{\rho}(v(\pi))(\mathbb{1} \otimes v(\rho))
\end{aligned}
$$

hold for all $\pi, \rho \in \hat{\mathcal{G}}$ and $b \in \mathcal{B}$.

3. Conversely, let $v(\pi) \in \mathcal{L}\left(\mathfrak{H}_{\pi}^{\prime}, \mathfrak{H}_{\pi}\right) \otimes \mathcal{B}, \pi \in \hat{\mathcal{G}}$, be a family of partial isometries for finitedimensional Hilbert spaces $\mathfrak{H}_{\pi}^{\prime}$ such that $v(\pi) v(\pi)^{*}=\gamma_{\pi}(\mathbb{1})$ holds for each $\pi \in \hat{\mathcal{G}}$. Then the following system $\left(\mathfrak{H}^{\prime}, \gamma^{\prime}, \omega^{\prime}\right)$ is a factor system of $(\mathcal{A}, \mathcal{G}, \alpha)$ :

$$
\begin{aligned}
& \gamma_{\pi}^{\prime}(b):=v(\pi)^{*} \gamma_{\pi}(b) v(\pi), \\
& \omega^{\prime}(\pi, \rho):=v(\pi \otimes \rho)^{*} \omega(\pi, \rho) \operatorname{id} \otimes \gamma_{\rho}(v(\pi))(\mathbb{1} \otimes v(\rho))
\end{aligned}
$$

for all $\pi, \rho \in \hat{\mathcal{G}}$ and $b \in \mathcal{B}$.

Proof. For sake of a concise notation we amplify all elements to a common domain specified by the context. Let $s(\pi) \in \mathcal{L}\left(\mathfrak{H}_{\pi}, V_{\pi}\right) \otimes \mathcal{A}, \pi \in \hat{\mathcal{G}}$, be the coisometries with $\pi \alpha(s(\pi))=s(\pi) \otimes \mathbb{1}_{\mathcal{G}}$ that generate the factor system $(\mathfrak{H}, \gamma, \omega)$.

1. Let $\pi, \rho$ be representations of $\hat{\mathcal{G}}$. Using the coisometry property of $s(\pi), s(\rho)$, and $s(\pi \otimes \rho)$ we obtain for the range and cokernel projection of $\omega(\pi, \rho)$

$$
\begin{aligned}
\omega(\pi, \rho) \omega(\pi, \rho)^{*} & =s(\pi \otimes \rho)^{*} s(\pi) s(\rho) s(\rho)^{*} s(\pi)^{*} s(\pi \otimes \rho) \\
& =s(\pi \otimes \rho)^{*} s(\pi \otimes \rho)=\gamma_{\pi \otimes \rho}(\mathbb{1}), \\
\omega(\pi, \rho)^{*} \omega(\pi, \rho) & =s(\rho)^{*} s(\pi)^{*} s(\pi \otimes \rho) s(\pi \otimes \rho)^{*} s(\pi) s(\rho) \\
& =s(\rho)^{*} s(\pi)^{*} s(\pi) s(\rho)=\gamma_{\rho}\left(\gamma_{\pi}(\mathbb{1})\right) .
\end{aligned}
$$

To show the other two asserted equations we compute the left and right hand side individually using the coisometry property and compare for all $b \in \mathcal{A}^{\mathcal{G}}$ :

$$
\begin{aligned}
& \omega(\pi, \rho) \gamma_{\rho}\left(\gamma_{\pi}(b)\right)=s(\pi \otimes \rho)^{*} s(\pi) s(\rho) s(\rho)^{*} s(\pi)^{*} b s(\pi) s(\rho) \\
& =s(\pi \otimes \rho)^{*} b s(\pi) s(\rho), \\
& \gamma_{\pi \otimes \rho}(b) \omega(\pi, \rho)=s(\pi \otimes \rho)^{*} b s(\pi \otimes \rho) s(\pi \otimes \rho)^{*} s(\pi) s(\rho) \\
& =s(\pi \otimes \rho)^{*} b s(\pi) s(\rho), \\
& \omega(\pi, \rho \otimes \sigma) \omega(\rho, \sigma)=s(\pi \otimes \rho \otimes \sigma)^{*} s(\pi) s(\rho \otimes \sigma) s(\rho \otimes \sigma)^{*} s(\rho) s(\sigma) \\
& =s(\pi \otimes \rho \otimes \sigma)^{*} s(\pi) s(\rho) s(\sigma), \\
& \omega(\pi \otimes \rho, \sigma) \gamma_{\sigma}(\omega(\pi, \rho))=s(\pi \otimes \rho \otimes \sigma)^{*} s(\pi \otimes \rho) s(\sigma) s(\sigma)^{*} s(\pi \otimes \rho)^{*} s(\pi) s(\rho) s(\sigma) \\
& =s(\pi \otimes \rho \otimes \sigma)^{*} s(\pi) s(\rho) s(\sigma) .
\end{aligned}
$$

2. Let $s^{\prime}(\pi) \in \mathcal{L}\left(\mathfrak{H}_{\pi}^{\prime}, V_{\pi}^{\prime}\right) \otimes \mathcal{A}, \pi \in \hat{\mathcal{G}}$, be the coisometries with $\pi \alpha\left(s^{\prime}(\pi)\right)=s^{\prime}(\pi) \otimes \mathbb{1}_{\mathcal{G}}$ that generate the factor system $\left(\mathfrak{H}^{\prime}, \gamma^{\prime}, \omega^{\prime}\right)$. Then the coisometry property implies that for each 
$\pi \in \hat{G}$ the element $v(\pi):=s(\pi)^{*} \cdot s^{\prime}(\pi)$ is a partial isometry satisfying $v(\pi) v(\pi)^{*}=s(\pi)^{*} s(\pi)=$ $\gamma_{\pi}(\mathbb{1})$ and $v(\pi)^{*} v(\pi)=s^{\prime}(\pi)^{*} s^{\prime}(\pi)=\gamma_{\pi}^{\prime}(\mathbb{1})$. Similarly the asserted relation of the *-homomorphisms $\gamma_{\pi}$ and $\gamma_{\pi}^{\prime}$ and of the elements $\omega(\pi, \rho)$ and $\omega^{\prime}(\pi, \rho)$ immediately follow from the coisometry property.

Next, we explain how the correspondence structure of the isotypic components of a free compact $\mathrm{C}^{*}$-dynamical system can be expressed only in terms of quantities of an associated factor system. For this purpose, let $(\mathfrak{H}, \gamma, \omega)$ be a factor system of a free compact $\mathrm{C}^{*}$-dynamical system $(\mathcal{A}, \mathcal{G}, \alpha)$ with fixed point algebra $\mathcal{B}$. Then, for a representation $(\pi, V)$ of $\mathcal{G}$, the left and right action of $\mathcal{B}$ and the inner product on $\Gamma(V)$ are given by

$$
\begin{aligned}
& b .(s(\pi) x)=s(\pi) \gamma_{\pi}(b) x, \\
& (s(\pi) x) \cdot b=s(\pi) x b, \\
& \langle s(\pi) x, s(\pi) y\rangle_{\mathcal{B}}=\left\langle x, \gamma_{\pi}\left(\mathbb{1}_{\mathcal{B}}\right) y\right\rangle_{\mathcal{B}}
\end{aligned}
$$

for all $b \in \mathcal{B}$ and $x, y \in \mathfrak{H}_{\pi} \otimes \mathcal{B}$. Moreover, for two representation $(\pi, V)$ and $(\rho, W)$ of $\mathcal{G}$ the multiplication map $m_{\pi, \rho}: \Gamma(V) \otimes_{\mathcal{B}} \Gamma(W) \rightarrow \Gamma(V \otimes W)$ can be written as

$$
m_{\pi, \rho}(s(\pi) x \otimes s(\rho) y)=s(\pi \otimes \rho) \omega(\pi, \rho) \gamma_{\rho}(x) y
$$

for all $x \in \mathfrak{H}_{\pi} \otimes \mathcal{B}$ and $y \in \mathfrak{H}_{\rho} \otimes \mathcal{B}$, where $\gamma_{\rho}(x) y$ is given by the linear extension of

$$
\gamma_{\rho}\left(v \otimes b_{1}\right)\left(w \otimes b_{2}\right)=v \otimes\left(\gamma_{\rho}\left(b_{1}\right)\left(w \otimes b_{2}\right)\right)
$$

for all $v \in \mathfrak{H}_{\pi}, w \in \mathfrak{H}_{\rho}$, and $b_{1}, b_{2} \in \mathcal{B}$. As a consequence, up to equivalence, the $\mathrm{C}^{*}$-dynamical system is uniquely determined by its factor system and vice versa. More precisely, we say that two factor systems $(\mathfrak{H}, \gamma, \omega)$ and $\left(\mathfrak{H}^{\prime}, \gamma^{\prime}, \omega^{\prime}\right)$ are conjugated if there is a family of partial isometries $v(\pi) \in \mathcal{L}\left(\mathfrak{H}_{\pi}^{\prime}, \mathfrak{H}_{\pi}\right) \otimes \mathcal{B}, \pi \in \hat{\mathcal{G}}$, satisfying the equations (4.4), (4.5), and (4.6) for all $\pi, \rho, \sigma \in \hat{\mathcal{G}}$ and $b \in \mathcal{B}$. Then we have the following 1-to-1 correspondence:

Theorem 4.4. Let $(\mathcal{A}, \mathcal{G}, \alpha)$ and $\left(\mathcal{A}^{\prime}, \mathcal{G}, \alpha^{\prime}\right)$ be free compact $\mathrm{C}^{*}$-dynamical systems with the same fixed point algebra $\mathcal{B}$ and let $(\mathfrak{H}, \gamma, \omega)$ and $\left(\mathfrak{H}^{\prime}, \gamma^{\prime}, \omega^{\prime}\right)$ be associated factor systems, respectively. Then the following statements are equivalent:

(a) The $\mathrm{C}^{*}$-dynamical systems $(\mathcal{A}, \mathcal{G}, \alpha)$ and $\left(\mathcal{A}^{\prime}, \mathcal{G}, \alpha^{\prime}\right)$ are equivalent.

(b) The factor systems $(\mathfrak{H}, \gamma, \omega)$ and $\left(\mathfrak{H}^{\prime}, \gamma^{\prime}, \omega^{\prime}\right)$ are conjugated.

Proof. As a distinction we add a prime to all notions referring to $\left(\mathcal{A}^{\prime}, \mathcal{G}, \alpha^{\prime}\right)$.

1. To prove that (a) implies (b) it suffices to show that for the same $\mathrm{C}^{*}$-dynamical system different choices of coisometries lead to conjugated factor systems. This is exactly the second statement of Lemma 4.3.

2. For the converse implication let $s(\pi) \in \mathcal{L}\left(\mathfrak{H}_{\pi}, V_{\pi}\right) \otimes \mathcal{A}, \pi \in \hat{\mathcal{G}}$, be the coisometries with $\pi_{13}$ id $\otimes \alpha(s(\pi))=s(\pi) \otimes \mathbb{1}_{\mathcal{G}}$ that generate the factor system $(\mathfrak{H}, \gamma, \omega)$, and likewise $s^{\prime}(\pi) \in$ $\mathcal{L}\left(\mathfrak{H}_{\pi}^{\prime}, V_{\pi}\right) \otimes \mathcal{A}$ for $\left(\mathfrak{H}^{\prime}, \gamma^{\prime}, \omega^{\prime}\right)$. Furthermore, let $v(\pi), \pi \in \hat{\mathcal{G}}$, be the partial isometries which realize the conjugation of the factor systems. Then a few moments thought show that, due to equations (4.4) and (4.5), for every representation $(\pi, V)$ of $\mathcal{G}$ the map

$$
\phi_{\pi}: \quad \Gamma^{\prime}(V) \rightarrow \Gamma(V), \quad s^{\prime}(\pi) x \mapsto s(\pi) v(\pi) x
$$

for all $x \in \mathfrak{H}_{\pi}^{\prime} \otimes \mathcal{B}$ is a well-defined isomorphism of correspondences of $\mathcal{B}$. Moreover, by equation (4.6), these isomorphisms intertwine the multiplication maps, i.e., we have

$$
m_{\pi, \rho}\left(\phi_{\pi}(x) \otimes \phi_{\rho}(y)\right)=\phi_{\pi \otimes \rho}\left(m_{\pi, \rho}^{\prime}(x \otimes y)\right)
$$


for all representations $(\pi, V),(\rho, W) \in \hat{\mathcal{G}}$ and all elements $x \in \Gamma^{\prime}(V)$ and $y \in \Gamma^{\prime}(W)$. Since $(\mathcal{A}, \mathcal{G}, \alpha)$ can be reconstructed from the correspondences $\Gamma(V)$ and the multiplicative structure between them (cf. Lemma 5.3 or [17, Section 2]), and likewise for $\left(\mathcal{A}^{\prime}, \mathcal{G}, \alpha^{\prime}\right)$ with $\Gamma^{\prime}(V)$, it is now easily checked that the maps $\phi_{\pi}, \pi \in \hat{\mathcal{G}}$, give rise to an equivalence between $(\mathcal{A}, \mathcal{G}, \alpha)$ and $\left(\mathcal{A}^{\prime}, \mathcal{G}, \alpha^{\prime}\right)$ (cf. also [28, Theorem 5]).

A particular simple class of free actions are so-called cleft actions (see [28]). Regarded as noncommutative principal bundles, these actions are characterized by the fact that all associated noncommutative vector bundles are trivial. For convenience of the reader we recall the definition.

Definition 4.5. A compact $\mathrm{C}^{*}$-dynamical system $(\mathcal{A}, \mathcal{G}, \alpha)$ is called cleft if for each irreducible representation $(\pi, V)$ of $\mathcal{G}$ the so-called generalized isotypic component

$$
A_{2}(\pi):=\left\{x \in \mathcal{L}(V) \otimes \mathcal{A} \mid \pi_{13} \text { id } \otimes \alpha(x)=x \otimes \mathbb{1}_{\mathcal{G}}\right\} \subseteq \mathcal{L}(V) \otimes \mathcal{A}
$$

contains a unitary element. It directly follows from Lemma 3.3 that cleft $\mathrm{C}^{*}$-dynamical systems are free.

Example 4.6. Given a unital $\mathrm{C}^{*}$-algebra $\mathcal{B}$ and a compact quantum group $\mathcal{G}$, the most basic example of a cleft action is given by the $\mathrm{C}^{*}$-dynamical system $(\mathcal{B} \otimes \mathcal{G}, \mathcal{G}$, id $\otimes \Delta)$. In fact, for any irreducible representation $(\pi, V)$ of $\mathcal{G}$ the unitary $U:=\pi_{13}^{*} \in \mathcal{L}(V) \otimes \mathcal{B} \otimes \mathcal{G}$ satisfies $\pi_{13}$ id $\otimes \Delta(U)=U \otimes \mathbb{1}_{\mathcal{G}}$.

Example 4.7. For $\mathcal{G}=\mathrm{SU}_{q}(2)$ the only cleft and ergodic action is the canonical action of $\mathrm{SU}_{q}(2)$ on itself (see [4, Corollary 5.9]). For $q=1$ this already follows from the seminal work of Wassermann [33].

Example 4.8 (cf. Example 3.6). For an arbitrary compact quantum group, the authors of [4] provide a classification of unitary fiber functors which preserve the dimension in terms of unitary 2-cocycles on the dual quantum group. It is not hard to see that the corresponding actions are cleft.

Example 4.9. It can be shown that the free $\mathrm{C}^{*}$-dynamical system $\left(\mathcal{A}\left(\mathbb{S}_{\theta^{\prime}}^{7}\right), \mathrm{SU}(2), \alpha\right)$ discussed in Example 3.5 is not cleft (cf. [15, Proposition 9]).

We continue with a characterization of cleft actions in terms of their factor systems. For this we recall that two projections $p \in \mathcal{L}(V) \otimes \mathcal{B}$ and $q \in \mathcal{L}(W) \otimes \mathcal{B}$ with finite-dimensional Hilbert spaces $V, W$ are called Murray-von Neumann equivalent over $\mathcal{B}$ if there is a partial isometry $v \in \mathcal{L}(V, W) \otimes \mathcal{B}$ satisfying $p=v^{*} v$ and $q=v v^{*}$.

Lemma 4.10. Let $(\mathcal{A}, \mathcal{G}, \alpha)$ be a free compact $\mathrm{C}^{*}$-dynamical system with fixed point algebra $\mathcal{B}$. Then the following statements are equivalent:

(a) The system $(\mathcal{A}, \mathcal{G}, \alpha)$ is cleft.

(b) For some and hence for every factor system $(\mathfrak{H}, \gamma, \omega)$ and every $(\pi, V) \in \hat{\mathcal{G}}$ the projection $\gamma_{\pi}\left(\mathbb{1}_{\mathcal{B}}\right)$ is Murray-von Neumann equivalent to $\mathbb{1}_{V} \otimes \mathbb{1}_{\mathcal{B}}$ over $\mathcal{B}$.

Proof. 1. If $(\mathcal{A}, \mathcal{G}, \alpha)$ is cleft, each $A_{2}(\pi), \pi \in \hat{\mathcal{G}}$, contains a unitary element $s(\pi)$. A factor system $(\mathfrak{H}, \gamma, \omega)$ is then given by $\mathfrak{H}_{\pi}=V_{\pi}$ and $\gamma_{\pi}(x)=s(\pi)^{*}(x \otimes \mathbb{1}) s(\pi)$ for all $\pi \in \hat{\mathcal{G}}$. In particular, we have $\gamma_{\pi}\left(\mathbb{1}_{\mathcal{B}}\right)=s(\pi)^{*} s(\pi)=\mathbb{1}_{V} \otimes \mathbb{1}_{\mathcal{B}}$. By Lemma 4.3 every other factor system $(\mathcal{A}, \mathcal{G}, \alpha)$ differs only by partial isometries in a respective amplification and therefore satisfies the same relation.

2. Conversely, suppose that $(\mathfrak{H}, \gamma, \omega)$ is a factor system of $(\mathcal{A}, \mathcal{G}, \alpha)$ such that for every $(\pi, V) \in \hat{G}$ the projections $\gamma_{\pi}\left(\mathbb{1}_{\mathcal{B}}\right)$ and $\mathbb{1}_{V} \otimes \mathbb{1}_{\mathcal{B}}$ are Murray-von Neumann equivalent. That 
is, we may find partial isometries $v(\pi) \in \mathcal{L}\left(V_{\pi}, \mathfrak{H}_{\pi}\right), \pi \in \hat{\mathcal{G}}$, such that $\gamma_{\pi}\left(\mathbb{1}_{\mathcal{B}}\right)=v(\pi) v(\pi)^{*}$ and $\mathbb{1}_{\pi} \otimes \mathbb{1}_{\mathcal{B}}=v(\pi)^{*} v(\pi)$. By conjugating the factor system with this family of partial isometries we may assume that $\mathfrak{H}_{\pi}=V_{\pi}$ and $\gamma_{\pi}\left(\mathbb{1}_{\mathcal{B}}\right)=\mathbb{1}_{\pi} \otimes \mathbb{1}_{\mathcal{B}}$. Moreover, for the factor system we may pick a family of coisometries $s(\pi) \in A_{2}(\pi), \pi \in \hat{\mathcal{G}}$, with $\gamma_{\pi}(b)=s(\pi)^{*}\left(\mathbb{1}_{\pi} \otimes b\right) s(\pi)$ for all $b \in \mathcal{B}$. Then we have $\mathbb{1}_{\pi} \otimes \mathbb{1}_{\mathcal{B}}=\gamma_{\pi}\left(\mathbb{1}_{\mathcal{B}}\right)=s(\pi)^{*} s(\pi)$, that is, $s(\pi)$ is unitary.

Example 4.11. Suppose we are in the context of Example 3.7 with $\mathcal{G}=\mathrm{SU}_{q}(2)$ and the natural representation $\left(\pi, \mathbb{C}^{2}\right)$ of $\mathrm{SU}_{q}(2)$. Furthermore, let $\mathcal{B}$ be the fixed point algebra of the induced free compact $\mathrm{C}^{*}$-dynamical system $\left(\mathcal{O}_{2}, \mathrm{SU}_{q}(2), \alpha\right)$. Then a few moments thought show that the ${ }^{*}$-homomorphism $\gamma_{\pi}: \mathcal{B} \rightarrow \mathcal{B}$ induced by the coisometry $s=\left(S_{1}^{*}, S_{2}^{*}\right)^{\top}$ satisfies $\gamma_{\pi}\left(\mathbb{1}_{\mathcal{B}}\right)=\mathbb{1}_{\mathcal{B}}$. Since [11, Proposition 6.10] implies that $K_{0}(\mathcal{B})$ can be identified with the integers in such a way that $\left[\mathbb{1}_{\mathcal{B}}\right]=1$ (see also $[12,14,16]$ ), it follows from Lemma 4.10 that $\left(\mathcal{O}_{2}, \mathrm{SU}_{q}(2), \alpha\right)$ is not cleft.

\section{Construction of free actions}

In the previous section we have seen that a free compact $\mathrm{C}^{*}$-dynamical system is uniquely determined by its factor system $(\mathfrak{H}, \gamma, \omega)$ and under which equivalence relation this becomes 1-to-1 correspondence (Theorem 4.4). In this section we will show that in fact every factor system $(\mathfrak{H}, \gamma, \omega)$ satisfying the algebraic relations of Lemma 4.3 gives rise to a free compact $\mathrm{C}^{*}$ dynamical system. The construction is based on the fact, that the factor system $(\mathfrak{H}, \gamma, \omega)$ allows us to completely reconstruct the correspondence structure of the multiplicity spaces $\Gamma(V)$ and their multiplicative structure, i.e., the factor system provides a unitary tensor functor $V \mapsto \Gamma(V)$ and hence a compact $\mathrm{C}^{*}$-dynamical system (see $[8,17]$ ). We recall the major steps in order to show that this construction yields a free compact $\mathrm{C}^{*}$-dynamical system with factor system $(\mathfrak{H}, \gamma, \omega)$.

Throughout the following let $\mathcal{B}$ be a fixed unital $\mathrm{C}^{*}$-algebra and let $\mathcal{G}$ be a fixed reduced compact quantum group. Furthermore, let $(\mathfrak{H}, \gamma, \omega)=\left(\mathfrak{H}_{\pi}, \gamma_{\pi}, \omega(\pi, \rho)\right)_{\pi, \rho \in \hat{\mathcal{G}}}$ be a family of finitedimensional Hilbert spaces $\mathfrak{H}_{\pi},{ }^{*}$-homomorphisms $\gamma_{\pi}: \mathcal{B} \rightarrow \mathcal{L}\left(\mathfrak{H}_{\pi}\right) \otimes \mathcal{B}$, and partial isometries $\omega(\pi, \rho) \in \mathcal{L}\left(\mathfrak{H}_{\pi} \otimes \mathfrak{H}_{\rho}, \mathfrak{H}_{\pi \otimes \rho}\right) \otimes \mathcal{B}$. By taking direct sums of irreducible representations, we define $\mathfrak{H}_{\pi}, \gamma_{\pi}$ and $\omega(\pi, \rho)$ for arbitrary representations $\pi, \rho$ of $\mathcal{G}$. In particular, for each intertwiner $T: V_{\pi} \rightarrow V_{\rho}$ we have a linear map $\mathfrak{H}(T): \mathfrak{H}_{\pi} \rightarrow \mathfrak{H}_{\rho}$.

Definition 5.1. A system $(\mathfrak{H}, \gamma, \omega)$ as described above is called a factor system for the pair $(\mathcal{B}, \mathcal{G})$ if it satisfies equations (4.1), (4.2), (4.3) for all $\pi, \rho \in \hat{\mathcal{G}}$ and $b \in \mathcal{B}$, and if the normalization conditions $\mathfrak{H}_{1}=\mathbb{C}, \gamma_{1}=\mathrm{id}_{\mathcal{B}}, \omega(1,1)=\mathbb{1}_{\mathcal{B}}$ holds.

From now on we suppose that $(\mathfrak{H}, \gamma, \omega)$ is a factor system. Then, for each representation $(\pi, V)$ of $\mathcal{G}$, we consider the vector space

$$
\Gamma(V):=\gamma_{\pi}(\mathbb{1})\left(\mathfrak{H}_{\pi} \otimes \mathcal{B}\right) .
$$

A few moments thought show that this space caries a natural right Hilbert $\mathcal{B}$-module structure given by restricting the action $\left(v_{1} \otimes b_{1}\right) . b_{2}:=v_{1} \otimes b_{1} b_{2}$ and the inner product $\left\langle v_{1} \otimes b_{1}, v_{2} \otimes b_{2}\right\rangle_{\mathcal{B}}:=$ $\left\langle v_{1}, v_{2}\right\rangle b_{1}^{*} b_{2}$ for $v_{1}, v_{2} \in \mathfrak{H}_{\pi}$ and $b_{1}, b_{2} \in \mathcal{B}$. Moreover, we equip $\Gamma(V)$ with the left action $b . x:=\gamma_{\pi}(b) x$ for $b \in \mathcal{B}$ and $x \in \Gamma(V)$. Then it is easily checked that $\Gamma(V)$ is a correspondence over $\mathcal{B}$ and that $V \mapsto \Gamma(V)$ becomes an additive functor from the representation category of $\mathcal{G}$ into the category of $\mathrm{C}^{*}$-correspondences over $\mathcal{B}$.

For each pair $(\pi, V),(\rho, W)$ of representation of $\mathcal{G}$ we define a linear map

$$
\begin{aligned}
m_{\pi, \rho}: & \Gamma(V) \otimes_{\mathcal{B}} \Gamma(W) \rightarrow \Gamma(V \otimes W), \\
& m_{\pi, \rho}(x \otimes y):=\omega(\pi, \rho) \gamma_{\rho}(x) y,
\end{aligned}
$$


where for elementary tensors we write briefly $\gamma_{\rho}\left(v \otimes b_{1}\right)\left(w \otimes b_{2}\right):=v \otimes \gamma_{\rho}\left(b_{1}\right)\left(w \otimes b_{2}\right)$ for all $v \in \mathfrak{H}_{\pi}, w \in \mathfrak{H}_{\rho}$, and $b_{1}, b_{2} \in \mathcal{B}$. It is easily checked that the maps $m_{\pi, \rho}$ are well-defined and behave naturally with respect to intertwiners. In fact, we are going to show that $V \mapsto \Gamma(V)$ together with the maps $m_{\pi, \rho}$ forms a unitary tensor functor in the sense of [17, Definition 2.1]. For convenience of the reader we recall the definition in the current context:

Definition 5.2. A linear functor $V \mapsto \Gamma(V)$ from the representation category of $\mathcal{G}$ into the category of $\mathrm{C}^{*}$-correspondences over $\mathcal{B}$ together with a $\mathcal{B}$-bilinear family of unitary maps

$$
m_{\pi, \rho}: \Gamma(V) \otimes_{\mathcal{B}} \Gamma(W) \rightarrow \Gamma(V \otimes W)
$$

for all representations $(\pi, V),(\rho, W)$ of $\mathcal{G}$ is called a unitary tensor functor if the following conditions hold:

1. For the trivial representation $(1, \mathbb{C}) \in \hat{G}$ we have $\Gamma(\mathbb{C})=\mathcal{B}$ and for all $(\pi, V) \in \hat{G}$ we have $m_{\pi, 1}(x \otimes b)=x . b$ and $m_{1, \pi}(b \otimes x)=b . x$ for all $x \in \Gamma(V), b \in \mathcal{B}$.

2. For every intertwiner $T: V \rightarrow W$ we have $\Gamma\left(T^{*}\right)=\Gamma(T)^{*}$.

3. The maps $m$ are associative in the sense that for all $\pi, \rho, \sigma \in \hat{G}$ we have

$$
m_{\pi, \rho \otimes \sigma} \circ\left(\mathrm{id} \otimes m_{\rho, \sigma}\right)=m_{\pi \otimes \rho, \sigma} \circ\left(m_{\pi, \rho} \otimes \mathrm{id}\right) .
$$

Lemma 5.3. The functor $V \mapsto \Gamma(V)$ and the maps $m_{\pi, \rho}: \Gamma(V) \otimes_{\mathcal{B}} \Gamma(W) \rightarrow \Gamma(V \otimes W)$ given by the equations (5.1) and (5.2), respectively, for $(\pi, V),(\rho, W) \in \hat{\mathcal{G}}$ constitute a unitary tensor functor.

Proof. 1. The normalization $\Gamma(\mathbb{C})=\mathcal{B}$ as correspondence immediately follows from $\mathfrak{H}_{1}=\mathbb{C}$ and $\gamma_{1}=\operatorname{id}_{\mathcal{B}}$. Moreover, the normalization $\omega(1,1)=1$ together with conditions (4.1) and (4.2) of the factor system imply $\omega(\pi, 1)=\mathbb{1}_{\mathfrak{H}_{\pi}}=\omega(1, \pi)$ that for every $(\pi, V) \in \hat{\mathcal{G}}$. Hence, we obtain $m_{\pi, 1}(x \otimes b)=\omega(\pi, 1) \gamma_{\mathcal{B}}(x) b=x . b$ and $m_{1, \pi}(b \otimes x)=\omega(1, \pi) \gamma_{\pi}(b) x=b . x$ for all $x \in \Gamma(V)$ and $b \in \mathcal{B}$.

2. For any intertwiner $T: V \rightarrow W$ it is easily checked that $\mathfrak{H}\left(T^{*}\right)=\mathfrak{H}(T)^{*}$ which in turn implies that $\Gamma(T)=\left.\mathfrak{H}(T) \otimes \operatorname{id}_{\mathcal{B}}\right|_{\Gamma(V)}$ is adjointable with $\Gamma(T)=\Gamma(T)^{*}$.

3. Associativity is an immediate consequence of the coaction and cocycle condition of the factor system. More precisely for all representations $\pi, \rho, \sigma \in \hat{\mathcal{G}}$ and elements $x \in \Gamma\left(V_{\pi}\right)$, $y \in \Gamma\left(V_{\rho}\right), z \in \Gamma\left(V_{\sigma}\right)$ we have

$$
\begin{aligned}
m_{\pi, \rho \otimes \sigma}\left(x \otimes m_{\rho, \sigma}(y \otimes z)\right)=\omega(\pi, \rho \otimes \sigma) \gamma_{\rho \otimes \sigma}(x)\left(\omega(\rho, \sigma) \gamma_{\sigma}(y) z\right) \\
\quad \stackrel{(4.2)}{=} \omega(\pi, \rho \otimes \sigma) \omega(\rho, \sigma) \gamma_{\sigma}\left(\gamma_{\rho}(x) y\right) z \stackrel{(4.3)}{=} \omega(\pi \otimes \rho, \sigma) \gamma_{\sigma}(\omega(\pi, \rho)) \gamma_{\sigma}\left(\gamma_{\rho}(x) y\right) z \\
\quad=\omega(\pi \otimes \rho, \sigma) \gamma_{\sigma}\left(\omega(\pi, \rho) \gamma_{\rho}(x) y\right) z=m_{\pi \otimes \rho, \sigma}\left(m_{\pi, \rho}(x \otimes y) \otimes z\right) .
\end{aligned}
$$

4. It remains to show that the maps $m_{\pi, \rho}: \Gamma(V) \otimes_{\mathcal{B}} \Gamma(W) \rightarrow \Gamma(V \otimes W)$ are unitary for all representations $(\pi, V),(\rho, W)$ of $\mathcal{G}$. To see that $m_{\pi, \rho}$ is isometric we observe that by equation (4.1) the projection $\omega(\pi, \rho)^{*} \omega(\pi, \rho)=\gamma_{\rho}\left(\gamma_{\pi}(\mathbb{1})\right)$ is larger than than the subspace of $\mathfrak{H}_{\pi} \otimes \mathfrak{H}_{\rho} \otimes \mathcal{B}$ generated by all $\gamma_{\rho}(x) y$ with $x \in \Gamma(V), y \in \Gamma(W)$. Hence, we have

$$
\begin{aligned}
& \left\langle m_{\pi, \rho}\left(x_{1} \otimes y_{1}\right), m_{\pi, \rho}\left(x_{2} \otimes y_{2}\right)\right\rangle_{\mathcal{B}}=\left\langle\gamma_{\rho}\left(x_{1}\right) y_{1}, \omega(\pi, \rho)^{*} \omega(\pi, \rho) \gamma_{\rho}\left(x_{2}\right) y_{1}\right\rangle_{\mathcal{B}} \\
& \quad=\left\langle\gamma_{\rho}\left(x_{1}\right) y_{1}, \gamma_{\rho}\left(x_{2}\right) y_{1}\right\rangle_{\mathcal{B}}=\left\langle x_{1} \otimes y_{1}, x_{2} \otimes y_{2}\right\rangle_{\mathcal{B}}
\end{aligned}
$$

for all $x_{1}, x_{2} \in \Gamma(V)$ and $y_{1}, y_{2} \in \Gamma(W)$. To show that $m_{\pi, \rho}$ is surjective, we notice that $\Gamma(V)$ is linearly generated by all elements of the form $\gamma_{\pi}(\mathbb{1})(v \otimes b)$ with $v \in \mathfrak{H}_{\pi}$ and $b \in \mathcal{B}$; and likewise for $\Gamma(W)$. By equation (4.1) the projection $\mathbb{1}_{\mathfrak{H}_{\pi}} \otimes \gamma_{\rho}\left(\mathbb{1}_{\mathcal{B}}\right)$ is larger than the cokernel projection 
$\omega(\pi, \rho)^{*} \omega(\pi, \rho)=\gamma_{\rho}\left(\gamma_{\pi}(\mathbb{1})\right)$. Choosing the elements $x:=v \otimes \mathbb{1}_{\mathcal{B}}$ and $y:=w \otimes b$, we therefore find that the range of $m_{\pi, \rho}$ contains all elements of the form

$$
\omega(\pi, \rho)\left(v \otimes \gamma_{\rho}(\mathbb{1})(w \otimes b)\right)=\omega(\pi, \rho)(v \otimes w \otimes b)
$$

with $v \in \mathfrak{H}_{\pi}, w \in \mathfrak{H}_{\rho}, b \in \mathcal{B}$. Hence, the image of $m_{\pi, \rho}$ contains the range of $\omega(\pi, \rho)$, which by equation $(4.1)$ is given by $\gamma_{\pi \otimes \rho}(\mathbb{1})\left(\mathfrak{H}_{\pi \otimes \rho} \otimes \mathcal{B}\right)=\Gamma(V \otimes W)$.

Having the unitary tensor functor in hands, we may construct a $\mathrm{C}^{*}$-dynamical system as presented in $[8,17]$. For convenience of the reader we briefly summarize the main steps. We consider the algebraic direct sum

$$
A:=\bigoplus_{(\pi, V) \in \hat{\mathcal{G}}} V \otimes \Gamma(\bar{V})
$$

We equip each summand of this space with its canonical $\mathcal{B}$-valued inner product given by $\langle v \otimes x, w \otimes y\rangle_{\mathcal{B}}=\langle v, w\rangle\langle x, y\rangle_{\mathcal{B}}$ for all $v, w \in V$ and $x, y \in \Gamma(\bar{V})$, and we extend the resulting inner product sesquilinearly to $A$. Moreover, we equip $A$ with the multiplication defined, for $\bar{v} \otimes x \in \bar{V} \otimes \Gamma(V)$ and $\bar{w} \otimes y \in \bar{W} \otimes \Gamma(W)$ with $(\pi, V),(\rho, W) \in \hat{\mathcal{G}}$, by the product

$$
(v \otimes x) \bullet(w \otimes y):=\sum_{k=1}^{N}\left(S_{k}^{*} \otimes \Gamma\left(\bar{S}_{k}\right)^{*}\right)\left(v \otimes w \otimes m_{\bar{\pi}, \bar{\rho}}(x \otimes y)\right) \in \sum_{k=1}^{N} V_{\sigma_{k}} \otimes \Gamma\left(\bar{V}_{\sigma_{k}}\right),
$$

where $S_{1}, \ldots, S_{N}$ is a complete set of isometric intertwiners $S_{k}: V_{\sigma_{k}} \rightarrow V \otimes W, \sigma_{k} \in \hat{\mathcal{G}}$, with respective conjugates $\bar{S}_{k}: \bar{V}_{\sigma_{k}} \rightarrow \bar{V} \otimes \bar{W}$. Extending this product bilinearly yields an associative multiplication on $A$. The algebra $\mathcal{B}$ can be regarded as the subalgebra of $A$ corresponding to the trivial representation, and the left and right module action of $\mathcal{B}$ coincides with the multiplication on $A$.

The next step is to construct an involution on $A$. For this purpose we first recall that for an irreducible representation $(\pi, V)$ of $\hat{G}$ there is a pair of intertwiners $R: \mathbb{C} \rightarrow V \otimes \bar{V}$ and $\bar{R}: \mathbb{C} \rightarrow \bar{V} \otimes V$ such that $\left(R^{*} \otimes \operatorname{id}_{V}\right)\left(\operatorname{id}_{V} \otimes \bar{R}\right)=\mathrm{id}_{V}$. With this we may define involutions ${ }^{+}: \Gamma(V) \rightarrow \Gamma(\bar{V})$ and ${ }^{+}: \bar{V} \rightarrow V$ by putting

$$
x^{+}:=m[x]^{*}\left(\Gamma(R)\left(\mathbb{1}_{\mathcal{B}}\right)\right), \quad \bar{v}^{+}:=i[\bar{v}]^{*} \bar{R}(1)
$$

where we briefly write $m[x]: \Gamma(\bar{V}) \rightarrow \Gamma(V \otimes \bar{V})$ for the map $m[x](y):=m_{\pi, \bar{\pi}}(x \otimes y)$ and $i[\bar{v}]: V \rightarrow V \otimes \bar{V}$ for the map $i[\bar{v}](w):=\bar{v} \otimes w$. Then for $\bar{v} \otimes x \in \bar{V} \otimes \Gamma(V) \subseteq A$ we may put $(\bar{v} \otimes x)^{+}:=\bar{v}^{+} \otimes x^{+}$and extend this anilinearly to a map on $A$. It can be shown that this involution turns $A$ into a ${ }^{*}$-algebra (see [17, Lemma 2.5]).

Remark 5.4. Our conventions for the inner products and the involution slightly deviate from [17], but the reader may easily adapt the arguments of [17] to our conventions.

Every summand $\bar{V} \otimes \Gamma(V)$ admits a unitary representation of $\mathcal{G}$ by acting on the first tensor factor. Taking direct sums yields a map $\alpha: A \rightarrow A \otimes \mathcal{G}$. This map is in fact a ${ }^{*}$-homomorphism satisfying $\left(\alpha \otimes \operatorname{id}_{\mathcal{G}}\right) \circ \alpha=(\alpha \otimes \Delta) \circ \alpha$ (see [17, Lemma 2.6]). Altogether we have an algebraic action of the quantum group $\mathcal{G}$ on the ${ }^{*}$-algebra $\mathcal{A}$. From this we may pass to a $\mathrm{C}^{*}$-dynamical system by taking the completion $\mathfrak{H}_{A}$ of $A$ with respect to the norm $\|x\|_{2}:=\left\|\langle x, x\rangle_{\mathcal{B}}\right\|^{1 / 2}$. Then the left multiplication of $A$ yields a faithful representation $\lambda: A \rightarrow \mathcal{L}\left(\mathfrak{H}_{A}\right)$ and a $\mathrm{C}^{*}$-algebra $\mathcal{A}:=\overline{\lambda(A)}$. The ${ }^{*}$-homomorphism $\alpha$ can be extended to an action $\alpha: \mathcal{A} \rightarrow \mathcal{A} \otimes \mathcal{G}$, which we denote by the same letter. Since we started with a unitary tensor functor, the corresponding compact $\mathrm{C}^{*}$-dynamical system $(\mathcal{A}, \mathcal{G}, \alpha)$ is free. For details we refer the reader to [8, Section 4]. 
Lemma 5.5. The free compact $\mathrm{C}^{*}$-dynamical system $(\mathcal{A}, \mathcal{G}, \alpha)$ admits $(\mathfrak{H}, \gamma, \omega)$ as one of its factor systems.

Proof. First note that for an irreducible representation $(\pi, V) \in \hat{\mathcal{G}}$, the $\pi$-isotypic component of $(\mathcal{A}, \mathcal{G}, \alpha)$ is obviously given by $V \otimes \Gamma(\bar{V})$. Hence the $\pi$-multiplicity space of the $\mathrm{C}^{*}$-dynamical system

$$
\Gamma_{\mathcal{A}}(V):=\left\{x \in V \otimes \mathcal{A} \mid \pi_{13} \mathrm{id}_{V} \otimes \alpha(x)=x \otimes \mathbb{1}_{\mathcal{G}}\right\} \subseteq V \otimes \bar{V} \otimes \Gamma(V)
$$

is isomorphic to $\Gamma(V)$ as a correspondence over $\mathcal{B}$. More precisely, a few moments thought show that an isomorphism is given by $\varphi: \Gamma(V) \rightarrow \Gamma_{\mathcal{A}}(V), x \mapsto R(1) \otimes x$. Next, fix an orthonormal basis $f_{1}, \ldots, f_{n}$ of $\mathfrak{H}_{\pi}$ and consider the elements

$$
s_{k}:=\gamma_{\pi}(\mathbb{1})\left(f_{k} \otimes \mathbb{1}_{\mathcal{B}}\right), \quad 1 \leq k \leq n .
$$

Then it is easily checked that the collection of elements $s_{k}(1 \leq k \leq n)$ for each $\pi \in \hat{\mathcal{G}}$ provide a factor system $(\tilde{\mathfrak{H}}, \tilde{\gamma}, \tilde{\omega})$ of $(\mathcal{A}, \mathcal{G}, \alpha)$ with Hilbert spaces $\tilde{\mathfrak{H}}_{\pi}=\mathfrak{H}_{\pi}$. In terms of the chosen basis $f_{1}, \ldots, f_{n}$, the ${ }^{*}$-homomorphism $\tilde{\gamma}_{\pi}: \mathcal{B} \rightarrow M_{n} \otimes \mathcal{B}$ for $\pi \in \hat{\mathcal{G}}$ is given by

$$
\tilde{\gamma}_{\pi}(b)_{i, j}=\left\langle\varphi\left(s_{i}\right), b . \varphi\left(s_{j}\right)\right\rangle_{\mathcal{B}}=\left\langle s_{i}, b . s_{j}\right\rangle_{\mathcal{B}}=\left\langle f_{i} \otimes \mathbb{1}_{\mathcal{B}}, \gamma_{\pi}(b)\left(f_{j} \otimes \mathbb{1}_{\mathcal{B}}\right)\right\rangle_{\mathcal{B}}=\gamma_{\pi}(b)_{i, j}
$$

for all $b \in \mathcal{B}$ (see Remark 4.2). That is, we have $\tilde{\gamma}=\gamma$ and similar computation shows that $\tilde{\omega}=\omega$, too. Consequently, we find that $(\mathfrak{H}, \gamma, \omega)$ is indeed a factor system of the free compact $\mathrm{C}^{*}$-dynamical system $(\mathcal{A}, \mathcal{G}, \alpha)$.

Summarizing the previous results, we have thus proved our main theorem:

Theorem 5.6. Let $\mathcal{B}$ be a unital $\mathrm{C}^{*}$-algebra and let $\mathcal{B}$ be a compact quantum group. Then there is a one-to-one correspondence between the set of equivalence classes of free $\mathrm{C}^{*}$-dynamical systems with fixed point algebra $\mathcal{B}$ and compact quantum group $\mathcal{G}$ and the set of conjugacy classes of factor systems for $(\mathcal{B}, \mathcal{G})$.

\section{Coverings of the noncommutative 2-torus}

Given a unital $\mathrm{C}^{*}$-algebra $\mathcal{B}$, we call a free compact $\mathrm{C}^{*}$-dynamical system $(\mathcal{A}, \mathcal{G}, \alpha)$ with a finite quantum group $\mathcal{G}$ and fixed point algebra $\mathcal{B}$ a finite covering of $\mathcal{B}$. The main purpose of this section is to use factor systems to show that finite coverings of generic irrational rotation $\mathrm{C}^{*}$-algebras are cleft (cf. Definition 4.5).

Lemma 6.1. Let $\theta \in \mathbb{R}$. Then every positive group homomorphism of $\mathbb{Z}+\theta \mathbb{Z}$ is a multiple of the identity.

Proof. Let $h: \mathbb{Z}+\theta \mathbb{Z} \rightarrow \mathbb{Z}+\theta \mathbb{Z}$ be a positive group homomorphism. Then for all $x, y \in \mathbb{Z}$ we have that $x+\theta y \geq 0$ implies $h(1) x+h(\theta) y \geq 0$ and $x+\theta y \leq 0$ implies $h(1) x+h(\theta) y \leq 0$. Considering $q:=-x / y$, it follows that for all $q \in \mathbb{Q}$ we have that $q \geq \theta$ implies $h(1) q \geq h(\theta)$ and $q \leq \theta$ implies $h(1) q \leq h(\theta)$. Taking the limit $q \rightarrow \theta$ in rationals, we may conclude that $h(1) \theta=h(\theta)$. Finally, for every $z=x+\theta y \in \mathbb{Z}+\theta \mathbb{Z}$ we obtain $h(z)=x h(1)+y h(\theta)=h(1) z$ as asserted.

Remark 6.2. Extending the preceding proof, the equation $h(1) \theta=h(\theta)$ is a quadratic equation with integer coefficients. Thence for non-quadratic $\theta$ the factor $h(1)$ must be a positive integer. 
Given a finite group $G$ and its representation $\operatorname{ring} R(G)$, it is a well-known fact that there is only one ring homomorphism $r: R(G) \rightarrow \mathbb{R}$ with $r(\pi)>0$ for every $\pi \in \hat{G}$, namely $r(\pi)=\operatorname{dim} \pi$ for every $\pi \in \hat{G}$. The next result shows that this statement remains true in the context of finite quantum groups.

Lemma 6.3. Let $\mathcal{G}$ be a finite quantum group and denote by $R(\mathcal{G})$ its representation ring. Then there is only one ring homomorphism $r: R(\mathcal{G}) \rightarrow \mathbb{R}$ with $r(\pi)>0$ for every $\pi \in \hat{\mathcal{G}}$, namely $r(\pi)=\operatorname{dim} \pi$ for every $\pi \in \hat{\mathcal{G}}$.

Proof. Let $r_{1}, r_{2}: R(\mathcal{G}) \rightarrow \mathbb{R}$ be two such positive, non-zero ring homomorphisms and let us fix $\pi \in \hat{\mathcal{G}}$. We consider the matrix $T(\pi)$ with rows and columns index by $\hat{\mathcal{G}}$ given by

$$
T(\pi)_{\rho, \sigma}:=\frac{m(\sigma, \rho \otimes \pi) r_{1}(\sigma)}{r_{1}(\rho \otimes \pi)}
$$

for all $\rho, \sigma \in \hat{\mathcal{G}}$, where $m(\sigma, \rho \otimes \pi)$ denotes the multiplicity of $\sigma$ in $\rho \otimes \pi$. A straightforward computation verifies that $T(\pi)$ is a stochastic matrix. Moreover, the vector $c=\left(c_{\rho}\right)_{\rho \in \hat{\mathcal{G}}}$ with $c_{\rho}:=r_{2}(\rho) / r_{1}(\rho)$ is an eigenvector of $T(\pi)$ with eigenvalue $\lambda=r_{2}(\pi) / r_{1}(\pi)$, because the homomorphism property implies

$$
(T(\pi) c)_{\rho}=\frac{1}{r_{1}(\rho \otimes \pi)} \sum_{\sigma \in \hat{\mathcal{G}}} m(\sigma, \rho \otimes \pi) r_{2}(\sigma)=\frac{r_{2}(\rho \otimes \pi)}{r_{1}(\rho \otimes \pi)}=\frac{r_{2}(\pi)}{r_{1}(\pi)} c_{\rho} .
$$

Since all eigenvalues of stochastic matrices lie in the unit disc, we now conclude that $r_{2}(\pi) \leq$ $r_{1}(\pi)$. Exchanging the role of $r_{1}$ and $r_{2}$ likewise yields $r_{2}(\pi) \leq r_{1}(\pi)$ and consequently we obtain $r_{1}=r_{2}$.

Theorem 6.4. Let $\theta \in \mathbb{R}$ be irrational and non-quadratic. Furthermore, let $\mathcal{G}$ be a finite quantum group. Then every free compact $\mathrm{C}^{*}$-dynamical system $(\mathcal{A}, \mathcal{G}, \alpha)$ with fixed point algebra $\mathcal{A}_{\theta}^{2}$ is cleft.

Proof. Let $(\mathcal{A}, \mathcal{G}, \alpha)$ be a free compact $\mathrm{C}^{*}$-dynamical system with $\mathcal{A}^{\mathcal{G}}=\mathcal{A}_{\theta}^{2}$ and let $(\mathfrak{H}, \gamma, \omega)$ be a factor system of $(\mathcal{A}, \mathcal{G}, \alpha)$. Then for every representation $\pi$ of $\mathcal{G}$ the ${ }^{*}$-homomorphism $\gamma_{\pi}: \mathcal{A}_{\theta}^{2} \rightarrow \mathcal{A}_{\theta}^{2} \otimes \mathcal{L}\left(\mathfrak{H}_{\pi}\right)$ induces a positive group homomorpism

$$
K_{0}\left(\gamma_{\pi}\right): \mathbb{Z}+\theta \mathbb{Z} \longrightarrow \mathbb{Z}+\theta \mathbb{Z},
$$

where we have identified $K_{0}\left(\mathcal{A}_{\theta}^{2} \otimes \mathcal{L}\left(\mathfrak{H}_{\pi}\right)\right)$ with $K_{0}\left(\mathcal{A}_{\theta}^{2}\right)=\mathbb{Z}+\theta \mathbb{Z}$. By Remark 6.2, this group homomorphism must be a positive integer of the identity, say for some factor $r(\pi)>0$. Given two representations $\pi, \rho$ of $\mathcal{G}$, we clearly have $r(\pi \oplus \rho)=r(\pi)+r(\rho)$. Moreover, the coaction condition of the factor system implies that $K_{0}\left(\gamma_{\rho}\right) \circ K_{0}\left(\gamma_{\pi}\right)=K_{0}\left(\gamma_{\pi \otimes \rho}\right)$ and therefore that $r(\rho) \cdot r(\pi)=$ $r(\pi \otimes \rho)$. As a consequence, we may extend the map $\pi \mapsto r(\pi)$ to a ring-homomorphism $r: R(\mathcal{G}) \rightarrow \mathbb{R}$. Lemma 6.3 then shows that $r(\pi)=\operatorname{dim}(\pi)$ holds for every $\pi \in \hat{\mathcal{G}}$ and hence we obtain

$$
\left[\gamma_{\pi}(\mathbb{1})\right]=K_{0}\left(\gamma_{\pi}\right)[\mathbb{1}]=r(\pi) \cdot[\mathbb{1}]=\operatorname{dim}(\pi) \cdot[\mathbb{1}]
$$

in $K_{0}\left(\mathcal{A}_{\theta}^{2}\right)$, i.e., the projections $\gamma_{\pi}(\mathbb{1})$ and $\mathbb{1}_{\pi} \otimes \mathbb{1}_{\mathcal{A}_{\theta}^{2}} \in \mathcal{L}\left(V_{\pi}\right) \otimes \mathcal{A}_{\theta}^{2}$ are stably equivalent. Since stable equivalence and Murray-von Neumann equivalence coincide for the $\mathrm{C}^{*}$-algebra $\mathcal{A}_{\theta}^{2}$ (see $[24,25]$ ), we finally conclude from Lemma 4.10 that $(\mathcal{A}, \mathcal{G}, \alpha)$ is cleft. 


\section{A Frames for Morita equivalence bimodules}

In this appendix we show that Morita equivalence bimodules between unital $\mathrm{C}^{*}$-algebras admit a so-called standard module frames. Although this might be well-known to experts, we have not found such a statement explicitly discussed in the literature.

Lemma A.1. Let $M$ be a Morita equivalence between unital $\mathrm{C}^{*}$-algebras $\mathcal{A}$ and $\mathcal{B}$. Then there are elements $x_{1}, \ldots, x_{n} \in M$ with

$$
\sum_{i=1}^{n} \mathcal{A}\left\langle x_{i}, x_{i}\right\rangle=\mathbb{1}
$$

In particular, for any collection of such elements we have a Fourier decomposition given for all $x \in M$ by

$$
x=\sum_{i=1}^{n} x_{k}\left\langle x_{k}, x\right\rangle_{\mathcal{B}} .
$$

Proof. The linear span of left inner products $J:={ }_{\mathcal{A}}\langle M, M\rangle$ is a dense ideal in $\mathcal{A}$. Since the invertible elements of $\mathcal{A}$ form an open subset, $J$ contains invertible elements and hence $J=\mathcal{A}$. That is, there are elements $x_{1}, \ldots, x_{n} \in M$ and $y_{1}, \ldots, y_{n} \in M$ with

$$
\mathbb{1}=\sum_{i=1}^{n} \mathcal{A}\left\langle x_{i}, y_{i}\right\rangle
$$

Then the Morita equivalence property implies

$$
y=\mathbb{1} \cdot y=\sum_{i=1}^{n}{ }_{\mathcal{A}}\left\langle x_{i}, y_{i}\right\rangle \cdot y=\sum_{i=1}^{n} x_{i} \cdot\left\langle y_{i}, y\right\rangle_{\mathcal{B}}
$$

for every $y \in M$. Now consider the matrix $Y \in \mathcal{B} \otimes M_{n}$ given by $Y_{i, j}:=\left\langle y_{i}, y_{j}\right\rangle_{\mathcal{B}}$ for $1 \leq i, j \leq n$. Since $Y$ is positive, we find a matrix $R=\left(R_{i, j}\right)_{i, j}$ in $\mathcal{B} \otimes M_{n}$ with $Y=R R^{*}$. Putting

$$
z_{k}:=\sum_{i=1}^{n} x_{i} \cdot R_{i, k}
$$

for all $1 \leq j \leq n$ we find

$$
\begin{aligned}
\sum_{k=1}^{n} \mathcal{A}\left\langle z_{k}, z_{k}\right\rangle & =\sum_{i, j, k=1}^{n} \mathcal{A}\left\langle x_{i} \cdot R_{i, k}, x_{j} . R_{j, k}\right\rangle=\sum_{i, j=1}^{n} \mathcal{A}\left\langle x_{i} \cdot\left(\sum_{k=1}^{n} R_{i, k} R_{j, k}^{*}\right), x_{j}\right\rangle \\
& =\sum_{i, j=1}^{n} \mathcal{A}\left\langle x_{i} \cdot\left\langle y_{i}, y_{j}\right\rangle_{\mathcal{B}}, x_{j}\right\rangle \stackrel{(\mathrm{A} .1)}{=} \sum_{j=1}^{n} \mathcal{A}\left\langle y_{j}, x_{j}\right\rangle=\mathbb{1} .
\end{aligned}
$$

\section{Acknowledgments}

We would like to acknowledge the Center of Excellence in Analysis and Dynamics Research (Academy of Finland, decision no. 271983 and no. 1138810) for supporting this research. The second name author also thanks the research fonds of the Department of Mathematics of the University of Hamburg. We would also like to express our greatest gratitude to the referees for providing very fruitful criticism. 


\section{References}

[1] Albeverio S., Høegh-Krohn R., Ergodic actions by compact groups on $C^{*}$-algebras, Math. Z. 174 (1980), $1-17$.

[2] Banica T., Fusion rules for representations of compact quantum groups, Exposition. Math. 17 (1999), 313337, math.QA/9811039.

[3] Baum P.F., De Commer K., Hajac P.M., Free actions of compact quantum group on unital C*-algebras, arXiv:1304.2812.

[4] Bichon J., De Rijdt A., Vaes S., Ergodic coactions with large multiplicity and monoidal equivalence of quantum groups, Comm. Math. Phys. 262 (2006), 703-728, math.QA/0502018.

[5] Connes A., Landi G., Noncommutative manifolds, the instanton algebra and isospectral deformations, Comm. Math. Phys. 221 (2001), 141-159, math.QA/0011194.

[6] De Commer K., Actions of compact quantum groups, arXiv:1604.00159.

[7] De Commer K., Yamashita M., A construction of finite index $\mathrm{C}^{*}$-algebra inclusions from free actions of compact quantum groups, Publ. Res. Inst. Math. Sci. 49 (2013), 709-735, arXiv:1201.4022.

[8] De Commer K., Yamashita M., Tannaka-Krein duality for compact quantum homogeneous spaces. I. General theory, Theory Appl. Categ. 28 (2013), no. 31, 1099-1138, arXiv:1211.6552.

[9] Echterhoff S., Nest R., Oyono-Oyono H., Principal non-commutative torus bundles, Proc. Lond. Math. Soc. 99 (2009), 1-31, arXiv:0810.0111.

[10] Ellwood D.A., A new characterisation of principal actions, J. Funct. Anal. 173 (2000), 49-60.

[11] Gabriel O., Fixed points of compact quantum groups actions on Cuntz algebras, Ann. Henri Poincaré 15 (2014), 1013-1036, arXiv:1210.5630.

[12] Gabriel O., Weber M., Fixed point algebras for easy quantum groups, SIGMA 12 (2016), 097, 21 pages, arXiv:1606.00569.

[13] Gootman E.C., Lazar A.J., Peligrad C., Spectra for compact group actions, J. Operator Theory 31 (1994), 381-399.

[14] Konishi Y., Nagisa M., Watatani Y., Some remarks on actions of compact matrix quantum groups on $C^{*}$-algebras, Pacific J. Math. 153 (1992), 119-127.

[15] Landi G., van Suijlekom W., Principal fibrations from noncommutative spheres, Comm. Math. Phys. 260 (2005), 203-225, math.QA/0410077.

[16] Marciniak M., Actions of compact quantum groups on $C^{*}$-algebras, Proc. Amer. Math. Soc. 126 (1998), 607-616.

[17] Neshveyev S., Duality theory for nonergodic actions, Münster J. Math. 7 (2014), 413-437, arXiv:1303.6207.

[18] Neshveyev S., Tuset L., Compact quantum groups and their representation categories, Cours Spécialisés, Vol. 20, Société Mathématique de France, Paris, 2013.

[19] Olesen D., Pedersen G.K., Takesaki M., Ergodic actions of compact abelian groups, J. Operator Theory 3 (1980), 237-269.

[20] Peligrad C., Locally compact group actions on $C^{*}$-algebras and compact subgroups, J. Funct. Anal. 76 (1988), 126-139.

[21] Phillips N.C., Equivariant $K$-theory and freeness of group actions on $C^{*}$-algebras, Lecture Notes in Math., Vol. 1274, Springer-Verlag, Berlin, 1987.

[22] Phillips N.C., Freeness of actions of finite groups on $C^{*}$-algebras, in Operator Structures and Dynamical Systems, Contemp. Math., Vol. 503, Amer. Math. Soc., Providence, RI, 2009, 217-257.

[23] Podleś P., Symmetries of quantum spaces. Subgroups and quotient spaces of quantum SU(2) and SO(3) groups, Comm. Math. Phys. 170 (1995), 1-20, hep-th/9402069.

[24] Rieffel M.A., The cancellation theorem for projective modules over irrational rotation $C^{*}$-algebras, Proc. London Math. Soc. 47 (1983), 285-302.

[25] Rieffel M.A., Nonstable $K$-theory and noncommutative tori, in Operator Algebras and Mathematical Physics (Iowa City, Iowa, 1985), Contemp. Math., Vol. 62, Amer. Math. Soc., Providence, RI, 1987, 267-279.

[26] Rieffel M.A., Proper actions of groups on $C^{*}$-algebras, in Mappings of Operator Algebras (Philadelphia, PA, 1988), Progr. Math., Vol. 84, Editors H. Araki, R.V. Kadison, Birkhäuser Boston, Boston, MA, 1990, $141-182$. 
[27] Schwieger K., Wagner S., Part I, Free actions of compact Abelian groups on $\mathrm{C}^{*}$-algebras, Adv. Math. 317 (2017), 224-266, arXiv:1505.00688.

[28] Schwieger K., Wagner S., Part II, Free actions of compact groups on $\mathrm{C}^{*}$-algebras, J. Noncommut. Geom. 11 (2017), 641-668, arXiv:1508.07904.

[29] Timmermann T., An invitation to quantum groups and duality. From Hopf algebras to multiplicative unitaries and beyond, EMS Textbooks in Mathematics, European Mathematical Society (EMS), Zürich, 2008.

[30] Wagner S., Free group actions from the viewpoint of dynamical systems, Münster J. Math. 5 (2012), 73-97, arXiv:1111.5562.

[31] Wassermann A., Ergodic actions of compact groups on operator algebras. I. General theory, Ann. of Math. 130 (1989), 273-319.

[32] Wassermann A., Ergodic actions of compact groups on operator algebras. II. Classification of full multiplicity ergodic actions, Canad. J. Math. 40 (1988), 1482-1527.

[33] Wassermann A., Ergodic actions of compact groups on operator algebras. III. Classification for SU(2), Invent. Math. 93 (1988), 309-354.

[34] Woronowicz S.L., Compact matrix pseudogroups, Comm. Math. Phys. 111 (1987), 613-665. 